\title{
The Kramers problem for SDEs driven by small, accelerated Lévy noise with exponentially light jumps
}

\author{
André de Oliveira Gomes * Michael A. Högele ${ }^{\dagger}$
}

\begin{abstract}
We establish Freidlin-Wentzell results for a nonlinear ordinary differential equation starting close to the stable state 0 , say, subject to a perturbation by a stochastic integral which is driven by an $\varepsilon$-small and $(1 / \varepsilon)$-accelerated Lévy process with exponentially light jumps. For this purpose we derive a large deviations principle for the stochastically perturbed system using the weak convergence approach developed by Budhiraja, Dupuis, Maroulas and collaborators in recent years. In the sequel we solve the associated asymptotic first escape problem from the bounded neighborhood of 0 in the limit as $\varepsilon \rightarrow 0$ which is also known as the Kramers problem in the literature.
\end{abstract}

Keywords: Freidlin-Wentzell theory; Large deviations principle; accelerated small noise Lévy diffusions; first passage times; first exit location; strongly tempered stable Lévy measure.

2010 Mathematical Subject Classification: 60H10; 60F10; 60J75; 60J05; 60E07.

\section{Introduction and main results}

\section{$1.1 \quad$ Introduction}

In this article we solve the Kramers problem for the family of strong solutions $\left(X^{\varepsilon}\right)_{\varepsilon>0}$ of the following stochastic differential equation (SDE for short)

$$
X_{t}^{\varepsilon}=x+\int_{0}^{t} b\left(X_{s}^{\varepsilon}\right) d s+\varepsilon \int_{0}^{t} \int_{\mathbb{R}^{d} \backslash\{0\}} G\left(X_{s-}^{\varepsilon}\right) z \tilde{N}^{\frac{1}{\varepsilon}}(d s, d z), \quad t \geqslant 0,
$$

on a neighborhood $D$ of 0 , which is the stable state of the underlying deterministic dynamical system $(\varepsilon=0)$. The driver of the stochastic perturbation $\tilde{N}^{\frac{1}{\varepsilon}}$ is a compensated Poisson random measure with compensator $d s \otimes \frac{1}{\varepsilon} \nu$, where $\nu$ is a Lévy measure satisfying a certain exponential integrability condition and the multiplicative coefficient $G$ being a non-vanishing scalar Lipschitz function. Our main result determines the asymptotic behavior $(\varepsilon \rightarrow 0)$ of the law and the expectation of the first exit time and location,

$$
\sigma^{\varepsilon}(x):=\inf \left\{t \geqslant 0 \mid X_{t}^{\varepsilon, x} \notin D\right\} \quad \text { and } \quad X_{\sigma^{\varepsilon}(x)}^{\varepsilon}, \text { respectively. }
$$

Our analysis relies on the establishment of a large deviations principle (LDP for short) based on the weak convergence approach, developed by Budhiraja, Dupuis, Maroulas and collaborators [13, 14]. Historically, the Kramers problem, that is, the escape time and location of a randomly excited deterministic dynamical system from close to a stable state at small intensity arose in the context of chemical reaction kinetics [3], 26] and [42. Nowadays this classical problem is virtually ubiquitous

\footnotetext{
*Departamento de Matemática Universidade Estadual de Campinas 13081-970 Campinas SP-Brazil; Institut für Mathematik Universität Potsdam; andrego@unicamp.br

†Departamento de matemáticas, Universidad de los Andes, Bogotá, Colombia; ma.hoegele@uniandes.edu.co
} 
and provides crucial insights in many diverse areas ranging from statistical mechanics, statistics, insurance mathematics, population dynamics and fluid dynamics to neurology. The mathematical theory of large deviations goes back to the seminal work by Crámer [19] before taking off in the seventies of the last century with the fundamental works by [23, 28, 29, 58. One main focus was the first exit problem for ordinary, delay and partial differential equations with small Gaussian noise in different settings and effects derived from it such as metastability and stochastic resonance. Classical texts with detailed expositions of the history of large deviations theory include [4, 5, 6, 9, 10, 11, 17, 21, 22, 24, 30, 55, 56. among others as well as the references therein. Furthermore, there is a lot of active research in the field, see for instance [18, 31, 45, 46, 54, 57. The major part of the body of literature studying large deviations and the Kramers law for stochastic differential equations with small noise is devoted to the study of Gaussian dynamics. For the dynamics of Markovian systems with jumps the literature is noticeably more fragmented, scattered and recent. It is due to the considerable variety of Lévy processes, including processes with heavy tails, and the resulting lack of moments, that there is no general large deviations theory for Lévy processes and diffusions with jumps. Large deviations results for certain classes of Lévy noises and Poisson random measures are given in [1, 7, 12, 27, 32, 44, 47, 50. and [59]. The first exit problem for small jump Lévy processes starts with the seminal paper by [33] for $\alpha$-stable processes and for more generally heavy-tailed processes by [20, 34, 36, 37, 49]. The mentioned works do not follow a large deviations regime in the sense of [13, 14] since the intensity measure of the underlying Lévy process is not rescaled by $\frac{1}{\varepsilon}$ as in our work. We mention [38, 39, where the authors provide in one dimension a complete scale study of asymptotic exit times as functions of $1 / \varepsilon$ ranging from polynomial via subexponential to exponential.

This article follows the rather different line of research started in [13] including not only an $\varepsilon$-dependent amplitude but also an $\varepsilon$-dependent acceleration of the jump intensity of the noise. It is this tuning between the jump size of order $\varepsilon$ and the intensity of $N^{\frac{1}{\varepsilon}}$ that permits to retrieve the large deviations regime for the dynamical system perturbed by a stochastic integral with respect to $\varepsilon \tilde{N}^{\frac{1}{\varepsilon}}$. In [13, 14] Budhiraja, Dupuis, Maroulas and collaborators derive large deviations results using new variational representation formulas for functionals of continuous time processes of this type. This has sparked a lot of ongoing research, cf. [15, 60, 61]. In [13] and in the recent monograph [16] the reader will find an extensive and up-to-date introduction to this subject.

The concise comprehension of the Kramers problem in this setting, which to our knowledge is missing in the literature to date, opens the door to deeper questions such as metastability, stochastic resonance and averaging, among other topics. The LDP of $\left(X^{\varepsilon, x}\right)_{\varepsilon>0}$ is given as an optimization problem under the dynamics solved by continuous controlled paths with a nonlocal component due to the pure jump noise.

For the derivation of the LDP we verify the sufficient abstract criteria established in [13. In their follow-up article [14 the authors apply this criteria to prove a large deviations result for a stochastic differential equation driven by small Lévy noise under the stricter assumption that the jumps of the noise component are bounded. There the authors use the same abstract sufficient criteria to establish a LDP for a stochastic partial differential equation driven by pure jump processes where the condition on the Lévy measure is relaxed (Condition 3.1 in [13]). In contrast to [14] our setting is the jump diffusion given by (1) with values in the finite dimensional Euclidean space $\mathbb{R}^{d}$. Our assumptions on the coefficients of (1) are rather standard monotonicity, Lipschitz and boundedness assumptions given in full detail in Subsection 1.2 and 1.4. They yield the LDP (first main theorem of this article) for a Lévy measure with infinite intensity allowing for unbounded jumps subject to an exponential integrability condition, such as in [14. However, the method to show the validity of the abstract sufficient criteria given in [13, 14 is different. The authors there base the construction of their weak convergence arguments only on a-priori bounds for the second moments of the jump infinite-dimensional jump diffusions. Naturally, the establishment of such a-priori bounds is difficult to obtain in the case of locally Lipschitz coefficients. The method we use to derive the large deviations result relies on the estimation of probabilities and on localization techniques based on a Bernstein-type inequality given in [25]. The use of localization techniques naturally allows the extension of the large deviations result obtained in this article to the case of locally Lipschitz coefficients, such as for instance the gradient case of a polynomial potential, which is clearly beyond the scope of this article.

Analogously to the classical Freidlin-Wentzell theory we solve the Kramers problem with a pseudo- 
potential given in terms of the good rate function of the LDP. In the Brownian case, under very mild assumptions on the coefficients of the SDE the respective controlled dynamics exhibits continuity properties that are crucial in the characterization of the first exit times. This differs strongly from the pure jump case which is the focus of this work. In this context, obtaining a closed form for the rate function is a hard task since the class of minimizers are scalar functions that represent shifts of the compensator of $\varepsilon \tilde{N}^{\frac{1}{\varepsilon}}$ on the nonlocal (possibly singular) component of the underlying controlled dynamics. This is an additional difficulty in the characterization of the first exit time in terms of the pseudo-potential. However, in the case of finite absolutely continuous jump measures we can solve the first exit time problem with the help of explicit formulas that we obtain for the controls. In other words, on an abstract level the physical intuition remains intact; however, since the control is given as a density w.r.t. the Lévy measure $\nu$, it is often hard to calculate the energy minimizing paths. This is the object of discussion in Section 1.4 where we illustrate our results with several examples. The first class for which we can solve everything explicitly in terms of the coefficients of (1) is the finite intensity benchmark case $\nu(d z)=e^{-|z|^{\beta}} d z, \beta \geqslant 2$. As a second example we introduce the natural class of Gauss-tempered $\alpha$-stable Lévy measures in the spirit of strongly tempered $\alpha$-stable Lévy measures studied in 52. For this class of measures we solve the Kramers problem subject to an additional continuity property for the controlled path dynamics (cf. Hypothesis G in Subsection 1.4). Lévy measures with compact support are another important class of measures that are covered in this setting.

Analogously to the Brownian case [22, 29] we construct for the lower bound of the first exit time a (modified) Markov chain approximation that takes into account the topological particularities of the Skorokhod space on which we have the LDP. In addition, the effect of the $(1 / \varepsilon)$-acceleration of the jump intensity enters as follows. The asymptotically exponentially negligible error estimates concerning the stickyness of the diffusion to its initial value, which in the classical Brownian case are valid for time intervals of order 1 , in our case only hold for time intervals of order $\varepsilon$.

The article is organized as follows. We start with the exposition of the generic setting followed by the discussion of the specific hypothesis for the LDP and the Kramers problem for finite intensity. It is followed by the previously mentioned Subsection 1.4 where we extend the results to infinite intensity, discuss the additional hypotheses and present natural classes of examples including the new class of Gauss-tempered $\alpha$-stable processes. In Section 2 we establish the LDP of $\left(X^{\varepsilon, x}\right)_{\varepsilon>0}$ given by (1) on a finite time interval. Section 3 deals with the upper and the lower bound of the Kramers problem. The appendix essentially contains auxiliary technical results for the derivation of LDP.

\subsection{The setting:}

The deterministic dynamics: Consider the following $\mathcal{C}^{1}$ vector field $b: \mathbb{R}^{d} \rightarrow \mathbb{R}^{d}, x \in \mathbb{R}^{d}$ and the deterministic dynamical system given as the solution flow $t \mapsto X_{t}^{0, x}$ of the ordinary differential equation

$$
\frac{d}{d t} X_{t}^{0, x}=b\left(X_{t}^{0, x}\right), \quad t \geqslant 0, \quad \text { and } \quad X_{0}^{0, x}=x
$$

subject to the following assumptions.

Hypothesis A. The vector field $b$ satisfies the following.

A.1: There is a constant $c_{1}>0$ such that

$$
\left\langle b\left(y_{1}\right)-b\left(y_{2}\right), y_{1}-y_{2}\right\rangle \leqslant-c_{1}\left|y_{1}-y_{2}\right|^{2}, \quad \text { for all } y_{1}, y_{2} \in \mathbb{R}^{d} \text {. }
$$

A.2: The point $0 \in \mathbb{R}^{d}$ is critical in that $b(0)=0$.

Remark 1. 1. It is well-known that under Hypothesis $\mathrm{A}$, for every initial point $x \in \mathbb{R}^{d}$ there is a unique solution $t \mapsto X_{t}^{0, x}$ of (2) for all $t \geqslant 0$. 
2. Hypothesis $\mathrm{\AA} 1$ implies that $D b(x)$ is strictly negative definite for all $x \in D$. In the case of a gradient system $b=-\nabla \mathcal{U}$ for some potential $\mathcal{U}: \mathbb{R}^{d} \rightarrow[0, \infty)$, this is equivalent to uniform convexity.

As a consequence, 0 is a hyperbolic stable fixed point of the dynamical system (2) in the sense that there is a constant $a>0$ such that all the eigenvalues $\lambda$ of $D^{2} b(0)$ have negative real part with $\Re(\lambda)<a<0$. Hence due to [51]-Theorem 5.1 it follows the limit $e^{a t} x(t)=0$.

In the sequel we define the stochastic perturbation $\varepsilon N^{\frac{1}{\varepsilon}}$ of (2) formally. See also [13] and [14].

The underlying noise $\varepsilon N^{\frac{1}{\varepsilon}}$. Let $\mathfrak{M}$ be the space of all locally finite measures defined on the Borel $\sigma$-algebra $\mathcal{B}\left(\mathbb{R}^{d} \backslash\{0\}\right)$.

We fix a non-atomic measure $\nu \in \mathfrak{M}$; that is, $\nu(\{z\})=0$ for all $z \in \mathbb{R}^{d}$ and $\nu(K)<\infty$ for every compact set $K \subset \mathbb{R}^{d}$ with $0 \notin K$. Theorem I.9.1 in [35] then shows that the measurable space $(\mathfrak{M}, \mathcal{B}(\mathfrak{M}))$ can be equipped with a unique non-atomic probability measure $\mathbb{P}$ such that the canonical map $N: \mathfrak{M} \rightarrow \mathfrak{M}, N(m):=m$ defines a Poisson random measure with intensity measure $d s \otimes \nu$ on $[0, \infty) \times \mathbb{R}^{d} \backslash\{0\}$, where $d s$ denotes the Lebesgue measure on the interval $[0, \infty)$. We also refer the reader to Proposition 19.4 in 53 . The compensated Poisson random measure of $N$ is defined by $\tilde{N}([0, s] \times A):=N([0, s] \times A)-s \nu(A)$ for all $s \geqslant 0$ and $A \in \mathcal{B}\left(\mathbb{R}^{d} \backslash\{0\}\right)$ such that $\nu(A)<\infty$. The expectation under $\mathbb{P}$ is denoted by $\mathbb{E}$. For all $\varepsilon>0$ we denote by $N^{\frac{1}{\varepsilon}}$ the Poisson random measure defined on the probability space $(\mathfrak{M}, \mathcal{B}(\mathfrak{M}), \mathbb{P})$ with intensity measure $d s \otimes \frac{1}{\varepsilon} \nu$ and its compensated counterpart $\tilde{N}^{\frac{1}{\varepsilon}}$. In particular, we have $N=N^{1}$ and $\tilde{N}=\tilde{N}^{1}$.

Consider the space $[0, \infty) \times \mathbb{R}^{d} \backslash\{0\} \times[0, \infty)$ and denote by $\overline{\mathfrak{M}}$ the space of the locally finite measures defined on the Borel $\sigma$-algebra $\mathcal{B}\left([0, \infty) \times \mathbb{R}^{d} \backslash\{0\} \times[0, \infty)\right)$. Analogously there is a unique probability measure $\overline{\mathbb{P}}$ defined on $(\overline{\mathfrak{M}}, \mathcal{B}(\overline{\mathfrak{M}}))$ such that the canonical map $\bar{N}: \overline{\mathfrak{M}} \rightarrow \overline{\mathfrak{M}}, \bar{N}(\bar{m}):=\bar{m}$, is a Poisson random measure on the probability space $(\overline{\mathfrak{M}}, \mathcal{B}(\overline{\mathfrak{M}}), \overline{\mathbb{P}})$ with intensity measure $d s \otimes \nu \otimes d r$, where $d r$ denotes the Lebesgue measure on the interval $[0, \infty)$. We write $\overline{\mathbb{E}}$ for the $\overline{\mathbb{P}}$ expectation.

Remark 2. For $(t, z, r) \in[0, \infty) \times \mathbb{R}^{d} \backslash\{0\} \times[0, \infty), t$ represents the time variable, $z$ the spatial jump increments $z$ of the underlying Lévy process associated to the Poisson random measure $\bar{N}$ and $r$ the frequency of the jump $z$ at time $t$.

For any $\varepsilon>0$ the Poisson random measure $N^{\frac{1}{\varepsilon}}$ has the following representation as a controlled random measure with respect to $\bar{N}$ under $\overline{\mathbb{P}}$. We have $\overline{\mathbb{P}}$-almost surely for every $t \geqslant 0$ and $A \in \mathcal{B}\left(\mathbb{R}^{d} \backslash\{0\}\right)$ the identity

$$
N^{\frac{1}{\varepsilon}}([0, t] \times A)=\int_{0}^{t} \int_{A} \int_{0}^{\infty} \mathbf{1}_{\left[0, \frac{1}{\varepsilon}\right]}(r) \bar{N}(d s, d z, d r) .
$$

For details we refer the reader to $[13$.

Hypothesis B. The measure $\nu \in \mathfrak{M}$ is non-atomic and satisfies the following conditions.

B.1: $\nu$ is a finite measure, $\nu\left(\mathbb{R}^{d} \backslash\{0\}\right)<\infty$.

B.2: There exists $\Gamma>0$ such that

$$
\int_{B_{1}^{c}(0)} e^{\Gamma|z|^{2}} \nu(d z)<\infty
$$

B.3: The measure $\nu$ is absolutely continuous with respect to the Lebesgue measure $d z$ on the measurable space $\left(\mathbb{R}^{d} \backslash\{0\}, \mathcal{B}\left(\mathbb{R}^{d} \backslash\{0\}\right)\right)$ and $\frac{d \nu}{d z}(z) \neq 0$ for every $z \in \mathbb{R}^{d} \backslash\{0\}$.

For a discussion of Hypothesis $\mathrm{B}$ we refer the reader to the remarks after the more general $\mathrm{Hy}$ pothesis E in Subsection 1.4

Remark 3. From Hypothesis B it follows for any $\varepsilon>0$ that the jumps of the stochastic process $L_{t}^{\varepsilon}=\int_{0}^{t} \int_{\mathbb{R}^{d} \backslash\{0\}} z \tilde{N}^{\frac{1}{\varepsilon}}(d s, d z)$ have finite intensity. 
The multiplicative coefficient. The function $G: \mathbb{R}^{d} \longrightarrow \mathbb{R} \backslash\{0\}$ satisfies the following.

Hypothesis C. There exists $L>0$ such that for all $x, y \in \mathbb{R}^{d}$ we have

$$
|G(x)-G(y)| \leqslant L|x-y| \quad \text { and } \quad|G(x)| \leqslant L(1+|x|) .
$$

The stochastic differential equation. Under Hypotheses $\mathrm{A}$ and $\mathrm{C}$ we consider for every $\varepsilon>0$ and $x \in \mathbb{R}^{d}$ the following SDE

$$
X_{t}^{\varepsilon, x}=x+\int_{0}^{t} b\left(X_{s}^{\varepsilon, x}\right) d s+\varepsilon \int_{0}^{t} \int_{\mathbb{R}^{d} \backslash\{0\}} G\left(X_{s-}^{\varepsilon, x}\right) z \tilde{N}^{\frac{1}{\varepsilon}}(d s, d z), \quad t \geqslant 0,
$$

We denote by $\left(\mathcal{F}_{t}\right)_{t \geqslant 0}$ the filtration given for any $t \geqslant 0$ by

$$
\mathcal{F}_{t}=\sigma\left(\left\{N^{1}([0, s] \times A \times C) \mid 0 \leqslant s \leqslant t, A \in \mathcal{B}\left(\mathbb{R}^{d} \backslash\{0\}\right), C \in \mathcal{B}([0, \infty))\right\} \vee \mathcal{N}\right), \quad t \geqslant 0,
$$

where $\mathcal{N}$ is the collection of the $\overline{\mathbb{P}}$-null sets in $\mathcal{B}\left([0, \infty) \times \mathbb{R}^{d} \backslash\{0\} \times[0, \infty)\right)$.

Let $\mathbb{D}\left([0, T], \mathbb{R}^{d}\right)$ be the linear space of càdlàg functions over the interval $[0, T], T>0$, with values in $\mathbb{R}^{d}$. It is well-known in the literature that the space $\mathbb{D}\left([0, T], \mathbb{R}^{d}\right)$ equipped with the topology generated by the $J_{1}$-metric $d_{J_{1}}$, known as the Skorokhod space, is a Polish space (see for instance Theorem 12.1 and Theorem 12.2 in [8]).

For the following result we cite Theorem IV-9.1 in Ikeda Watanabe and Theorem 6.4.5 of [2].

Theorem 1. Given $\varepsilon, T>0, x \in \mathbb{R}^{d}$ and $\nu \in \mathfrak{M}$ let Hypotheses $\mathbb{A}, \mathbb{B}$ and $\square$ be satisfied. Then there is

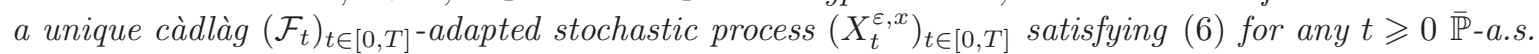
In addition, $X^{\varepsilon, x}=\left(X_{t}^{\varepsilon, x}\right)_{t \in[0, T]}$ is a strong Markov process with respect to the filtration $\left(\mathcal{F}_{t}\right)_{t \in[0, T]}$. In particular, due to the uniqueness of the strong solution of (6) in the sense of Definition IV-1.5 in [35] there is a $\overline{\mathbb{P}}$-a.s. well-defined measurable map $\mathcal{G}^{\varepsilon, x}: \overline{\mathcal{M}} \longrightarrow \mathbb{D}\left([0, T] ; \mathbb{R}^{d}\right)$ such that $\varepsilon N^{\frac{1}{\varepsilon}} \mapsto X^{\varepsilon, x}$.

\subsection{Statement of the main results}

Let the standing assumptions of Subsection 1.2 be satisfied, in particular Hypotheses $\mathrm{A} B \mathrm{~B}$ and $\mathrm{C}$

\subsubsection{A LDP for $\left(X^{\varepsilon, x}\right)_{\varepsilon>0}$}

Whenever possible without confusion we shall drop the index for the initial condition $x$. In this paragraph we fix some notation and introduce the necessary objects for the statement of the LDP of $\left(X^{\varepsilon, x}\right)_{\varepsilon>0}$ following [13] and [14. For fixed $T>0$ and a measurable function $g:[0, T] \times \mathbb{R}^{d} \backslash\{0\} \rightarrow$ $[0, \infty)$ we define the entropy functional by

$$
\mathcal{E}_{T}(g):=\int_{0}^{T} \int_{\mathbb{R}^{d} \backslash\{0\}}(g(s, z) \ln g(s, z)-g(s, z)+1) \nu(d z) d s .
$$

For every $M \geqslant 0$ we define the sublevel sets of the functional $\mathcal{E}_{T}$ by

$$
\mathfrak{S}^{M}:=\left\{g:[0, T] \times \mathbb{R}^{d} \backslash\{0\} \longrightarrow[0, \infty) \mathrm{mb} \mid \mathcal{E}_{T}(g) \leqslant M\right\} \quad \text { and set } \quad \mathfrak{S}:=\bigcup_{M \geqslant 0} \mathfrak{S}^{M} .
$$

Given $T>0, x \in \mathbb{R}^{d}$ and $g \in \mathfrak{S}$ we consider the controlled integral equation

$$
U^{g}(t ; x)=x+\int_{0}^{t} b\left(U^{g}(s ; x)\right) d s+\int_{0}^{t} \int_{\mathbb{R}^{d} \backslash\{0\}} G\left(U^{g}(s ; x)\right)(g(s, z)-1) z \nu(d z) d s, t \in[0, T] .
$$

It is standard in the literature (see Theorem 3.7 in [14]) that the equation (9) has a unique solution $U^{g} \in C\left([0, T], \mathbb{R}^{d}\right)$ and it satisfies the uniform bound

$$
\sup _{t \in[0, T]} \sup _{g \in \mathfrak{S}^{M}}\left|U^{g}(t ; x)\right|<\infty \quad \text { for all } M>0 .
$$


In particular, the map $\mathcal{G}^{0, x}: \mathfrak{S} \rightarrow C\left([0, T], \mathbb{R}^{d}\right), g \mapsto \mathcal{G}^{0, x}(g):=U^{g}(\cdot ; x)$ is well-defined for any fixed $x \in \mathbb{R}^{d}$. For $\varphi \in C\left([0, T], \mathbb{R}^{d}\right)$ we define $\mathfrak{S}_{\varphi, x}:=\left\{g \in \mathfrak{S} \mid \varphi=\mathcal{G}^{0, x}(g)\right\}$ the preimage of $\varphi$ under $\mathcal{G}^{0, x}$ and set $\mathbb{J}_{x, T}: \mathbb{D}\left([0, T], \mathbb{R}^{d}\right) \rightarrow[0, \infty]$

$$
\mathbb{J}_{x, T}(\varphi):=\inf _{g \in \mathfrak{S}_{\varphi, x}} \mathcal{E}_{T}(g),
$$

with the convention that $\inf \emptyset=\infty$.

Theorem 2. Let Hypotheses $A, B$ and $\square$ be satisfied for some $\nu \in \mathfrak{M}, T>0$ and $x \in D$ fixed and let $X^{\varepsilon, x}=\left(X_{t}^{\varepsilon, x}\right)_{t \in[0, T]}, \varepsilon>0$, be the strong solution of (6) given in Theorem 11. Then the family $\left(X^{\varepsilon, x}\right)_{\varepsilon>0}$ satisfies a LDP with the good rate function $\mathbb{J}_{x, T}$ given by (11) in the Skorokhod space $\mathbb{D}\left([0, T], \mathbb{R}^{d}\right)$. This means that, for any $a \geqslant 0$ the sublevel set $\left\{\mathbb{J}_{x, T} \leqslant a\right\}$ is compact in $\mathbb{D}\left([0, T], \mathbb{R}^{d}\right)$ and for any $G \subset \mathbb{D}\left([0, T], \mathbb{R}^{d}\right)$ open and $F \subset \mathbb{D}\left([0, T], \mathbb{R}^{d}\right)$ closed,

$$
\begin{aligned}
& \liminf _{\varepsilon \rightarrow 0} \varepsilon \ln \overline{\mathbb{P}}\left(X^{\varepsilon, x} \in G\right) \geqslant-\inf _{\varphi \in G} \mathbb{J}_{x, T}(\varphi) \text { and } \\
& \limsup _{\varepsilon \rightarrow 0} \varepsilon \ln \overline{\mathbb{P}}\left(X^{\varepsilon, x} \in F\right) \leqslant-\inf _{\varphi \in F} \mathbb{J}_{x, T}(\varphi) .
\end{aligned}
$$

\subsubsection{The asymptotic first exit problem of $X^{\varepsilon, x}$ from $D$ as $\varepsilon \rightarrow 0$}

We make the additional assumptions as follows.

Hypothesis D. Let us consider a bounded domain $D \subset \mathbb{R}^{d}$ with $0 \in D, \partial D \in \mathcal{C}^{1}$ and that $b$ is inward-pointing on $\partial D$, that is,

$$
\langle b(z), n(z)\rangle<0, \quad \text { for all } z \in \partial D,
$$

where the vector field $\partial D \ni z \mapsto n(z) \in \mathbb{R}^{d}$ denotes the outer normal on $\partial D$.

Remark 4. The first statement of Hypothesis $\mathrm{D}$ implies that the solution of (2) is positive invariant on $\bar{D}$, that is, for all $x \in \bar{D}$, we have $X_{t}^{0, x} \in D$ for all $t \geqslant 0$ and $X_{t}^{0, x} \rightarrow 0$ as $t \rightarrow \infty$.

Given $\varepsilon>0, x \in D$ and $\nu \in \mathfrak{M}$ satisfying Hypotheses $\mathrm{A}$, B $\mathrm{C}$ and $\mathrm{D}$ we define the first exit time of the solution $X^{\varepsilon, x}$ of (6) from $D$

$$
\sigma^{\varepsilon}(x):=\inf \left\{t \geqslant 0 \mid X_{t}^{\varepsilon, x} \notin D\right\}
$$

and the first exit location $X_{\sigma^{\varepsilon}(x)}^{\varepsilon, x}$.

The function $V$ quantifying the cost of shifting the intensity jump measure by a scalar control $g$ and steering $U^{g}(t ; x)$ from its initial position $x$ to some $z \in \mathbb{R}^{d}$ in cheapest time is defined as

$$
V(x, z):=\inf _{T>0} \inf \left\{\mathbb{J}_{x, T}(\varphi) \mid \varphi \in \mathbb{D}\left([0, T], \mathbb{R}^{d}\right): \varphi(T)=z\right\} \quad \text { for } x, z \in \mathbb{R}^{d} .
$$

The function $V(0, z)$ is called the quasi-potential of the stable state 0 with potential height

$$
\bar{V}:=\inf _{z \notin D} V(0, z) .
$$

We are ready to present our main result. The proof is the combination of Corollary 18, Theorem 5 , Theorem [6] and Remark 24 in Section 3

Theorem 3. Let Hypotheses $A, B, \square$ and $D$ be satisfied. Then $\bar{V}<\infty$ and we obtain the following result.

1. For any $x \in D$ and $\delta>0$, we have

$$
\lim _{\varepsilon \rightarrow 0} \overline{\mathbb{P}}\left(e^{\frac{\bar{v}-\delta}{\varepsilon}}<\sigma^{\varepsilon}(x)<e^{\frac{\bar{V}+\delta}{\varepsilon}}\right)=1 .
$$

Furthermore, for all $x \in D$ it follows $\lim _{\varepsilon \rightarrow 0} \varepsilon \ln \overline{\mathbb{E}}\left[\sigma^{\varepsilon}(x)\right]=\bar{V}$. 
2. For any closed set $F \subset D^{c}$ satisfying $\inf _{z \in F} V(0, z)>\bar{V}$ and any $x \in D$, we have

$$
\lim _{\varepsilon \rightarrow 0} \overline{\mathbb{P}}\left(X_{\sigma^{\varepsilon}(x)}^{\varepsilon, x} \in F\right)=0 .
$$

In particular, if $\bar{V}$ is taken by a unique point $z^{*} \in D^{c}$, it follows, for any $x \in D$ and $\delta>0$, that

$$
\lim _{\varepsilon \rightarrow 0} \overline{\mathbb{P}}\left(\left|X_{\sigma^{\varepsilon}(x)}^{\varepsilon, x}-z^{*}\right|<\delta\right)=1 .
$$

\subsection{Extensions and remarks}

In this subsection we discuss the analogous statements of Theorem 2 and Theorem 3 in a general framework with a vector-valued function $G: \mathbb{R}^{d} \times \mathbb{R}^{d} \longrightarrow \mathbb{R}^{d}$ and a Lévy measure $\nu$ with infinite intensity. It is the aim of this subsection to present a sufficient condition for the LDP and the solution of the Kramers problem of the following family of processes $\left(X^{\varepsilon, x}\right)_{\varepsilon>0}$. Such a condition is given below as Hypothesis $G$ and is formulated as a continuity property for the controlled dynamics. This turns out to be hard to be verified in general and needs to be studied case by case.

For every $\varepsilon>0$ and $x \in D$ we consider the unique strong solution $\left(X_{t}^{\varepsilon, x}\right)_{t \geqslant 0}$ of

$$
X_{t}^{\varepsilon, x}=x+\int_{0}^{t} b\left(X_{s}^{\varepsilon, x}\right) d s+\int_{0}^{t} \int_{\mathbb{R}^{d} \backslash\{0\}} G\left(X_{s-}^{\varepsilon, x}, z\right) \varepsilon \tilde{N}^{\frac{1}{\varepsilon}}(d s, d z), \quad t \geqslant 0 .
$$

The function $b$ remains unchanged satisfying Hypothesis $\mathrm{A}$ and $\mathrm{D}$ For every $\varepsilon>0 \varepsilon \tilde{N}^{\frac{1}{\varepsilon}}$ is a compensated Poisson random measure defined on $(\mathfrak{M}, \mathcal{B}(\mathfrak{M}), \overline{\bar{P}})$ with compensator given by $d s \otimes \frac{1}{\varepsilon} \nu(d z)$ where $\nu \in \mathfrak{M}$ satisfies the following assumption which replaces Hypothesis $\mathbb{B}$.

Hypothesis E. The measure $\nu \in \mathfrak{M}$ is non-atomic and satisfies the following conditions.

E.1: The measure $\nu$ is a Lévy measure, i.e. $\int_{\mathbb{R}^{d} \backslash\{0\}}\left(1 \wedge|z|^{2}\right) \nu(d z)<\infty$.

E.2: $\nu \in \mathfrak{M}$ satisfies $\int_{B_{1}^{c}(0)} e^{\Gamma|z|^{2}} \nu(d z)<\infty$ for some $\Gamma>0$.

E.3: There are positive constants $p, q$ such that

$$
\limsup _{\varepsilon \rightarrow 0+}|\ln (\varepsilon)|^{p} \cdot \int_{|z| \leqslant \sqrt{\varepsilon}|\ln (\varepsilon)|^{q}}|z|^{2} \nu(d z)<\infty .
$$

Remark 5. 1. We stress that Hypothesis $\mathrm{E}$ is weaker than Hypothesis $\mathrm{B}$ and covers a wide class of Lévy measures. The measure $\nu(d z)=e^{-|z|^{\beta}} d z, \beta \geqslant 2$, is an important benchmark case and is the model case covered by Hypothesis B In the literature it is known as a super-exponentially light jump measure.

2. More generally Hypothesis E covers a class of Lévy measures which mimics the strongly tempered $\alpha$-stable measures introduced by Rosiński [52, however, with a Gaussian damping term in order to guarantee the moment condition in Hypothesis B.2 (and Hypothesis E.2 resp.). We define a Gauss-tempered $\alpha$-stable Lévy measure $\nu$ as follows. For the radial coordinate $r=|z|$, for some measure $R \in \mathfrak{M}, \gamma>0$ and $\alpha \in(0,2)$ satisfying $\int_{\mathbb{R}^{d} \backslash\{0\}}|z|^{\alpha} R(d z)<\infty$ we set

$$
\nu(A):=\int_{\mathbb{R}^{d} \backslash\{0\}} \int_{0}^{\infty} \mathbf{1}_{A}(r z) \frac{e^{-\frac{\gamma}{2} r^{2}}}{r^{\alpha+1}} d r R(d z), \quad A \in \mathcal{B}\left(\mathbb{R}^{d} \backslash\{0\}\right) .
$$

3. Hypothesis $\mathrm{E}$ is satisfied by Lévy measures with compact support as in the seminal paper [13]. 
4. Hypothesis $\mathrm{E} 3$ is a non-degeneracy condition on the pole of the Lévy measure in 0 . It is trivially satisfied for finite Lévy measures (Hypothesis B.1). In addition, it is satisfied for any Gauss-tempered $\alpha$-stable Lévy measure $\nu$ and any $\alpha \in(0,2)$ as defined in (20)

$$
\begin{aligned}
\int_{|z| \leqslant \sqrt{\varepsilon}|\ln (\varepsilon)|^{q}}|z|^{2} \nu(d z) & =\int_{\mathbb{R}^{d} \backslash\{0\}} \int_{0}^{\infty}|r v|^{2} \mathbf{1}\left\{|r v| \leqslant \sqrt{\varepsilon}|\ln (\varepsilon)|^{q}\right\} \frac{e^{-\frac{\gamma}{2} r^{2}}}{r^{\alpha+1}} d r R(d v) \\
& \leqslant \int_{\mathbb{R}^{d} \backslash\{0\}}|v|^{2} \int_{0}^{\infty}|r|^{1-\alpha} \mathbf{1}\left\{|r| \leqslant \frac{\sqrt{\varepsilon}|\ln (\varepsilon)|^{q}}{|v|}\right\} d r R(d v) \\
& =\int_{\mathbb{R}^{d} \backslash\{0\}}|v|^{2} \frac{1}{2-\alpha}\left(\frac{\sqrt{\varepsilon}|\ln (\varepsilon)|^{q}}{|v|}\right)^{2-\alpha} R(d v) \\
& =\frac{\left(\sqrt{\varepsilon}|\ln (\varepsilon)|^{q}\right)^{2-\alpha}}{2-\alpha} \int_{\mathbb{R}^{d} \backslash\{0\}}|v|^{\alpha} R(d v) .
\end{aligned}
$$

The expression on the right-hand side is finite by definition and tends to 0 as $\varepsilon \rightarrow 0$ with polynomial rate and consequently satisfies the relation (19).

The vector-valued multiplicative coefficient $G: \mathbb{R}^{d} \times \mathbb{R}^{d} \rightarrow \mathbb{R}^{d}$ satisfies the following assumption that replaces Hypothesis $\mathrm{C}$.

Hypothesis $\mathbf{F}$. There exists $L>0$ such that for all $x, y, z \in \mathbb{R}^{d}$ we have

$$
|G(x, z)-G(y, z)| \leqslant L|z||x-y| \quad \text { and } \quad|G(x, z)| \leqslant L|z|(1+|x|) .
$$

Remark 6. Under Hypotheses $\mathrm{A}, \mathrm{E}$ (which covers B) and $\mathrm{E}$ (which covers $\mathrm{C}$ ) the statements of Theorem 1 remain valid.

The following hypothesis is the fundamental assumption of this subsection.

Hypothesis G. For every $\rho_{0}>0$ there exist a constant $M>0$ and a non-decreasing function $\xi$ : $\left[0, \rho_{0}\right] \rightarrow \mathbb{R}^{+}$with $\lim _{\rho \rightarrow 0} \xi(\rho)=0$ satisfying the following. For all $x_{0}, y_{0} \in \mathbb{R}^{d}$ such that $\left|x_{0}-y_{0}\right| \leqslant \rho_{0}$ there exist $\Phi \in C\left(\left[0, \xi\left(\rho_{0}\right)\right], \mathbb{R}^{d}\right)$ and $g \in \mathfrak{S}^{M}$ such that $\Phi\left(\xi\left(\rho_{0}\right)\right)=y_{0}$ and solving

$$
\Phi(t)=x_{0}+\int_{0}^{t} b(\Phi(s)) d s+\int_{0}^{t} \int_{\mathbb{R}^{d} \backslash\{0\}} G(\Phi(s), z)(g(s, z)-1) \nu(d z) d s, \quad t \in\left[0, \xi\left(\rho_{0}\right)\right] .
$$

Remark 7. Hypothesis G is covered under the assumptions stated in Theorem 3 This is proved in Proposition 17.

Construction of the good rate function and the potential. Let Hypotheses $\mathrm{A}, \mathrm{D}, \mathrm{E}, \mathrm{F}$ and $\mathrm{G}$ be satisfied. For fixed $x \in \mathbb{R}^{d}, T>0$, define $\mathcal{E}_{T}$ by (77) and $\mathfrak{S}$ by $(8)$. Let $\mathcal{G}^{0, x}: \mathfrak{S} \longrightarrow C\left([0, T] ; \mathbb{R}^{d}\right)$ defined as $\mathcal{G}^{0, x}(g):=U^{g}(\cdot ; x)$ where the function $U^{g}$ is the unique solution of the following controlled integral equation

$$
U^{g}(t ; x)=x+\int_{0}^{t} b\left(U^{g}(s ; x)\right) d s+\int_{0}^{t} \int_{\mathbb{R}^{d} \backslash\{0\}} G\left(U^{g}(s ; x), z\right)(g(s, z)-1) \nu(d z) d s, t \in[0, T] .
$$

With this notation the definition of the good rate function $\mathbb{J}_{x, T}$ in (11) and the potential height $\bar{V}$ in (14) remain unchanged and the results of Theorem 2 and Theorem 3 are carried over in the next theorem.

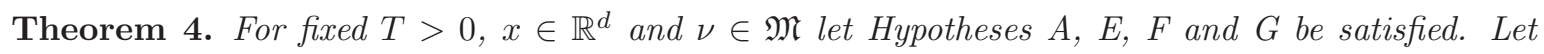
$\left(X^{\varepsilon, x}\right)_{\varepsilon>0}$ be the family of strong solutions of (18). Then we have the following.

1. The family $\left(X^{\varepsilon, x}\right)_{\varepsilon>0}$ satisfies a LDP in $\mathbb{D}\left([0, T], \mathbb{R}^{d}\right)$ with the good rate function $\mathbb{J}_{x, T}$ given by (11). 
2. For any domain $D \subset \mathbb{R}^{d}$ satisfying Hypothesis $D$ and $x \in D$ the results (15)-(17) of Theorem 3 hold for the respective exit time $\sigma^{\varepsilon}(x)$.

In this setting the proof of the preceding results is virtually identical to the proofs of Theorem 2 and Theorem 3 and is therefore omitted.

Remark 8. We comment on the assumptions stated on Hypothesis B and further generalizations made on Hypothesis $\mathrm{E}$ for the Lévy measure $\nu$.

1. The exponential integrability assumption of Hypothesis $\mathrm{B} 2$ (and analogously $\mathrm{E} 2$ ) is sufficient to verify the abstract condition for a LDP in [13] as explained in the introduction. More precisely, it is used in the proof of auxiliary a-priori bounds for the controlled integrals stated in Lemma 9 , It is proved under the general Hypothesis E In particular, the exponential Young inequality applied there seems near optimal and hard to relax. On the other hand even to derive a LDP for one dimensional empirical means of i.i.d. random variables some exponential integrability of the underlying law is necessary. See Lemma 2.2.5 (b) in the proof of the Cramér Theorem 2.3.3 in 22 .

2. A priori, the extension from Hypothesis B to Hypothesis E bears the difficulty, that it is hard to solve the control problem (40) which under Hypotheses B.1 and B. 3 can be solved explicitly in Proposition 16. As a consequence we state its solvability in Hypothesis $\mathrm{G}$ as a proper hypothesis, since its solution may require rather different techniques to be verified in particular situations.

\section{The large deviations principle}

In this section we prove Theorem 2 Let Hypotheses $\mathrm{A}$ B and $\mathrm{C}$ be satisfied for some $\nu \in \mathfrak{M}$. For every $\varepsilon>0, T>0$ and $x \in \mathbb{R}^{d}$, we consider the strong solution $\left(X^{\varepsilon, x}\right)_{t \in[0, T]}$ of the SDE (6). By Theorem 1 the map

$$
\mathcal{G}^{\varepsilon, x}: \mathfrak{M} \rightarrow \mathbb{D}\left([0, T], \mathbb{R}^{d}\right), \quad \mathcal{G}^{\varepsilon, x}\left(\varepsilon N^{\frac{1}{\varepsilon}}\right):=X^{\varepsilon, x},
$$

is measurable with respect to the Borel sigma algebras associated to the vague convergence topology in $\mathfrak{M}$ and the (Skorokhod) $J_{1}$ topology in $\mathbb{D}\left([0, T], \mathbb{R}^{d}\right)$. On the other hand, given $g \in \mathfrak{S}$, the wellposedness of the integral equation (9) yields the existence of a measurable map

$$
\mathcal{G}^{0, x}: \mathfrak{S} \rightarrow C\left([0, T], \mathbb{R}^{d}\right), \quad \mathcal{G}^{0, x}(g):=U^{g}(\cdot, x) .
$$

The main task in the proof of Theorem 2 is the verification of the two statements of Condition 2.2 in 14 for $\left(\mathcal{G}^{\varepsilon, x}\right)_{\varepsilon>0}$ and $\mathcal{G}^{0, x}$, which combined imply the LDP (cf. Theorem 2.4 in [14]). We essentially follow the notation introduced in [13] and [14].

\subsection{The weak convergence approach}

Notation. Denote by $\overline{\mathcal{P}}$ the predictable $\sigma$-field on $[0, T] \times \overline{\mathfrak{M}}$ with respect to the filtration $\left(\mathcal{F}_{t}\right)_{t \in[0, T]}$. We define the space of positive (random) controls in $\overline{\mathfrak{M}}$

$$
\overline{\mathfrak{A}}^{+}:=\left\{\varphi:[0, T] \times \mathbb{R}^{d} \backslash\{0\} \times \overline{\mathfrak{M}} \rightarrow[0, \infty) \mid \varphi \text { is }\left(\overline{\mathcal{P}} \otimes \mathcal{B}\left(\mathbb{R}^{d} \backslash\{0\}\right), \mathcal{B}([0, \infty))\right) \text { - measurable }\right\} .
$$

Given a covering of $\mathbb{R}^{d} \backslash\{0\}$ by compact sets $\left(K_{n}\right)_{n \in \mathbb{N}}$ we define the set of the $n$-cutoff positive (random) controls

$$
\overline{\mathfrak{A}}_{b, n}^{+}:=\left\{\varphi \in \overline{\mathfrak{A}}^{+} \mid \varphi(t, x, \bar{m})\left\{\begin{array}{ll}
\in\left[\frac{1}{n}, n\right], & x \in K_{n}, \\
=1, & x \in K_{n}^{c},
\end{array} \text { for all }(t, \bar{m}) \in[0, T] \times \overline{\mathfrak{M}}\right\} .\right.
$$

The set of positive bounded controls is then given by

$$
\overline{\mathfrak{A}}_{b}^{+}:=\bigcup_{n \in \mathbb{N}} \overline{\mathfrak{A}}_{b, n}^{+} \quad \text { and } \quad \mathfrak{U}_{+}^{M}:=\left\{\varphi \in \overline{\mathfrak{A}}_{b}^{+}: \varphi(., ., \bar{m}) \in \mathfrak{S}^{M} \quad \overline{\mathbb{P}}-\text { a.s }\right\}, \quad M>0,
$$


is the set of positive bounded random controls whose entropy functional is $\overline{\mathbb{P}}$-a.s. bounded by $M$. We associate to every $g \in \mathfrak{S}^{M}$ the measure

$$
\mathcal{B}\left([0, T] \times \mathbb{R}^{d} \backslash\{0\}\right) \ni A \mapsto \nu_{T}^{g}(A):=\int_{A} g(s, z) \nu(d z) d s
$$

and identify $\mathfrak{S}^{M}$ with the space of associated measures $\left\{\nu_{T}^{g} \mid g \in \mathfrak{S}^{M}\right\} \subset \mathfrak{M}$ equipped with the topology induced by the vague convergence on $\mathfrak{M}$. We refer the reader to Lemma 5.1 in [14] which ensures that this identity produces a topology in $\mathfrak{S}^{M}$ under which $\mathfrak{S}^{M}$ turns out to be compact.

For any fixed $M>0$ and a family $\left(\varphi_{\varepsilon}\right)_{\varepsilon>0} \subset \mathfrak{U}_{+}^{M}$ we set $\psi_{\varepsilon}:=\frac{1}{\varphi_{\varepsilon}}$. The random measure $N^{\frac{\varphi_{\varepsilon}}{\varepsilon}}$ is a controlled random measure given by

$$
N^{\frac{\varphi_{\varepsilon}}{\varepsilon}}([0, t] \times U):=\int_{0}^{t} \int_{U} \int_{0}^{\infty} \mathbf{1}_{\left[0, \frac{\varphi_{\varepsilon}}{\varepsilon}(s, z)\right]}(r) \bar{N}(d s, d z, d r) \quad \text { for all } t \in[0, T], U \in \mathcal{B}\left(\mathbb{R}^{d} \backslash\{0\}\right) .
$$

Recall that the canonical map $\bar{N}: \mathfrak{M} \rightarrow \mathfrak{M}, \bar{N}(\bar{m}):=\bar{m}$ is the Poisson random measure defined on $(\overline{\mathfrak{M}}, \mathcal{B}(\overline{\mathfrak{M}}), \overline{\mathbb{P}})$ with intensity measure $d s \otimes \nu \otimes d r$.

Since $\varphi_{\varepsilon} \in \mathfrak{U}_{+}^{M}$ yields that $\varphi_{\varepsilon}$ is bounded from below and above on a compact set in $[0, T] \times \mathbb{R}^{d} \backslash\{0\}$ and $\varphi_{\varepsilon}=1$ outside of that compact, we can use Girsanov's theorem in the form of Lemma 2.3 in [13]. Therefore the Doleans-Dade exponential of $\psi_{\varepsilon}$ with respect to $\bar{N}$ under $\overline{\mathbb{P}}$ defined for any $t \in[0, T]$ by

$$
\mathfrak{E}\left(\psi_{\varepsilon}\right)(t):=\exp \left(\int_{0}^{t} \int_{\mathbb{R}^{d} \backslash\{0\}} \int_{0}^{\frac{1}{\varepsilon}} \ln \psi_{\varepsilon}(s, z) \bar{N}(d s, d z, d r)+\int_{0}^{t} \int_{\mathbb{R}^{d} \backslash\{0\}} \int_{0}^{\frac{1}{\varepsilon}}\left(-\psi_{\varepsilon}(s, z)+1\right) d r \nu(d z) d s\right)
$$

is an $\left(\mathcal{F}_{t}\right)_{t \in[0, T]}$ - martingale under $\overline{\mathbb{P}}$. In addition, the measure

$$
\mathcal{B}(\overline{\mathfrak{M}}) \ni G \mapsto \mathbb{Q}_{T}^{\varepsilon}(G):=\int_{G} \mathfrak{E}\left(\psi_{\varepsilon}\right)(T) d \overline{\mathbb{P}}
$$

is a probability measure on $(\overline{\mathfrak{M}}, \mathcal{B}(\overline{\mathfrak{M}}))$. Furthermore, the measures $\overline{\mathbb{P}}$ and $\mathbb{Q}_{T}^{\varepsilon}$ are mutually absolutely continuous and the controlled random measure $\varepsilon N^{\frac{1}{\varepsilon}} \varphi_{\varepsilon}$ under $\mathbb{Q}_{T}^{\varepsilon}$ has the same law as $\varepsilon N^{\frac{1}{\varepsilon}}$ under $\overline{\mathbb{P}}$ on $(\overline{\mathfrak{M}}, \mathcal{B}(\overline{\mathfrak{M}}))$. For more details we refer the reader to Lemma 2.3 in 13 and further references given there. Denote by $\tilde{X}^{\varepsilon, x}:=\mathcal{G}^{\varepsilon, x}\left(\varepsilon N^{\frac{\varphi_{\varepsilon}}{\varepsilon}}\right)$ the unique strong solution of the following controlled SDE

$$
\tilde{X}_{t}^{\varepsilon, x}=x+\int_{0}^{t} b\left(\tilde{X}_{s}^{\varepsilon, x}\right) d s+\int_{0}^{t} \int_{\mathbb{R}^{d} \backslash\{0\}} G\left(\tilde{X}_{s-}^{\varepsilon, x}\right) z\left(\varepsilon N^{\frac{\varphi_{\varepsilon}}{\varepsilon}}(d s, d z)-\nu(d z) d s\right), \quad t \in[0, T] .
$$

Technical estimates: The following lemma is crucial in the proof of Theorem 2 and the reader can find its proof in the Subsection 4.1 of the appendix.

Lemma 9. Let $\nu \in \mathfrak{M}$ satisfy Hypothesis $\mathbb{E}$. Then for any $M, T>0$ and $x \in \mathbb{R}^{d}$ we have the following statements

$$
\begin{aligned}
& \sup _{g \in \mathfrak{S}^{M}} \iint_{[0, T] \times \mathbb{R}^{d} \backslash\{0\}}|z|^{2} g(s, z) \nu(d z) d s<\infty, \\
& \sup _{g \in \mathfrak{S}^{M}} \iint_{[0, T] \times \mathbb{R}^{d} \backslash\{0\}}|z||g(s, z)-1| \nu(d z) d s<\infty \quad \text { and } \\
& \lim _{\delta \rightarrow 0} \sup _{g \in \mathfrak{S}^{M}} \sup _{\substack{0 \leqslant t<t^{\prime} \leqslant T \\
\left|t-t^{\prime}\right| \leqslant \delta}} \iint_{\left[t, t^{\prime}\right] \times \mathbb{R}^{d} \backslash\{0\}}|z||g(s, z)-1| \nu(d z) d s=0 .
\end{aligned}
$$

The process $\tilde{X}^{\varepsilon, x}$ introduced in (23) has the following localization property used in the sequel. 


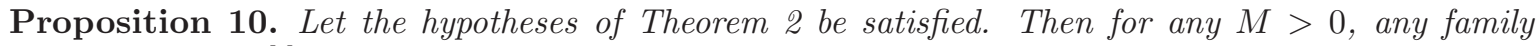
$\left(\varphi_{\varepsilon}\right)_{\varepsilon>0}, \varphi_{\varepsilon} \in \mathfrak{U}_{+}^{M}$, any function $\mathcal{R}:(0,1] \rightarrow(0, \infty)$ satisfying the limits $\lim _{\varepsilon \rightarrow 0+} \mathcal{R}(\varepsilon)=\infty$ and $\lim _{\varepsilon \rightarrow 0+} \varepsilon \mathcal{R}^{2}(\varepsilon)=0, x \in \mathbb{R}^{d}$ and $T>0$ we have the following. There exist constants $\varepsilon_{0} \in(0,1]$ and $C>0$ such that $\varepsilon \in\left(0, \varepsilon_{0}\right]$ implies

$$
\overline{\mathbb{P}}\left(\sup _{s \in[0, T]}\left|\tilde{X}_{s}^{\varepsilon, x}\right|>\mathcal{R}(\varepsilon)\right) \leqslant 2 e^{-\frac{1}{2} \mathcal{R}(\varepsilon)}+C \varepsilon \mathcal{R}(\varepsilon) .
$$

The proof uses an exponential Bernstein-type inequality for martingales from [25] and given in Subsection 4.1 of the appendix.

For any $\varepsilon>0$ and $\mathcal{R}:(0,1] \longrightarrow(0, \infty)$ satisfying the limits $\mathcal{R}(\varepsilon) \rightarrow \infty$ and $\varepsilon \mathcal{R}^{2}(\varepsilon) \rightarrow 0$ as $\varepsilon \rightarrow 0$, such as in the statement of Proposition [10, let us define the $\left(\mathcal{F}_{t}\right)_{t \in[0, T] \text {-stopping time }}$

$$
\tilde{\tau}_{\mathcal{R}(\varepsilon)}^{\varepsilon}:=\inf \left\{t \geqslant 0 \mid \tilde{X}_{t}^{\varepsilon, x} \notin B_{\mathcal{R}(\varepsilon)}(0)\right\} \wedge T .
$$

Proposition 11. For any $\varepsilon>0, x \in \mathbb{R}^{d}$ and $\mathcal{R}:(0,1] \longrightarrow(0, \infty)$ given as in Proposition 10 the following holds. There exists $\Lambda:=\Lambda(L, M, T, x)>0$ and $\varepsilon_{1}>0$ such that

$$
\sup _{\varepsilon \in\left(0, \varepsilon_{1}\right]} \overline{\mathbb{E}}\left[\sup _{t \in\left[0, \tilde{\tau}_{\mathcal{R}(\varepsilon)}^{\varepsilon}\right]}\left|\tilde{X}_{t}^{\varepsilon, x}\right|^{2}\right] \leqslant \Lambda .
$$

The preceding estimate is proved in Subsection 4.1 of the appendix.

\subsection{Proof of Theorem 2}

The following proposition is a continuity statement of the map $\mathcal{G}^{0, x}$ for any $x \in \mathbb{R}^{d}$.

Proposition 12. For every $x \in \mathbb{R}^{d}, M \geqslant 0$ and $n \in \mathbb{N}$ let $g_{n}, g \in \mathfrak{S}^{M}$ such that $\nu_{T}^{g_{n}} \rightarrow \nu_{T}^{g}$ in the vague topology of $\mathfrak{S}$ as $n \rightarrow \infty$. Then there exists a subsequence $\left(g_{n_{k}}\right)_{k \in \mathbb{N}} \subset\left(g_{n}\right)_{n \in \mathbb{N}}$ such that

$$
\mathcal{G}^{0, x}\left(g_{n_{k}}\right) \rightarrow \mathcal{G}^{0, x}(g), \quad \text { as } k \rightarrow \infty
$$

in the uniform topology of $C\left([0, T], \mathbb{R}^{d}\right)$.

Proof. For convenience we drop the dependence on the parameter $x \in \mathbb{R}^{d}$ in what follows. We set $U_{n}:=U^{g_{n}}=\mathcal{G}^{0}\left(g_{n}\right)$. Estimate (10) yields a constant $K>0$ such that

$$
\sup _{n \in \mathbb{N}} \sup _{t \in[0, T]}\left|U_{n}(t ; x)\right| \leqslant K .
$$

Due to (26) it follows

$$
\lim _{\delta \rightarrow 0} \sup _{n \in \mathbb{N}} \sup _{|t-s| \leqslant \delta}\left|U_{n}(t ; x)-U_{n}(s ; x)\right|=0,
$$

which implies that $\left(U_{n}\right)_{n \in \mathbb{N}}$ is a family of equicontinuous uniformly bounded functions in $C\left([0, T], \mathbb{R}^{d}\right)$. The Arzelà-Ascoli compactness theorem yields a limit in the uniform topology $U \in C\left([0, T], \mathbb{R}^{d}\right)$ for some subsequence $\left(g_{n_{k}}\right)_{k \in \mathbb{N}} \subset\left(g_{n}\right)_{n \in \mathbb{N}}$. By the uniform estimate (30), the continuity of the functions $b$ and $G$ and (24) dominated convergence yields

$$
U(t ; x)=x+\int_{0}^{t} b(U(s ; x)) \mathrm{d} s+\int_{0}^{t} \int_{\mathbb{R}^{d} \backslash\{0\}} G(U(s ; x))(g(s, z)-1) z \nu(\mathrm{d} z) \mathrm{d} s \quad \text { for all } t \in[0, T] .
$$

The uniqueness of solution of (9) implies that $U=U^{g}=\mathcal{G}^{0}(g)$.

For $\varepsilon>0, x \in \mathbb{R}^{d}, M \geqslant 0$ and $\varphi \in \mathfrak{U}_{+}^{M}$ the following result is a weak law of large numbers type of statement for the measurable maps $\mathcal{G}^{\varepsilon, x}$ under the action of the controlled random measures $\varepsilon \tilde{N}^{\frac{\varphi}{\varepsilon}}$. 
Proposition 13. Given $M \geqslant 0$ let $\varphi \in \mathfrak{U}_{+}^{M}$ and $\left(\varphi_{\varepsilon}\right)_{\varepsilon>0} \subset \mathfrak{U}_{+}^{M}$ such that $\varphi_{\varepsilon} \Rightarrow \varphi$ in law as $\varepsilon \rightarrow 0$. Then for all $x \in \mathbb{R}^{d} \mathcal{G}^{0, x}(g)$ is a limit point in law of $\mathcal{G}^{\varepsilon, x}\left(\varepsilon N^{\frac{\varphi_{\varepsilon}}{\varepsilon}}\right)$ in $\mathbb{D}\left([0, T], \mathbb{R}^{d}\right)$.

Proof. We drop the dependence on $x$ of $\tilde{X}^{\varepsilon, x}$ and for every $\varepsilon \in(0,1]$ and $t \in[0, T]$ we define

$$
\begin{aligned}
J_{t}^{\varepsilon} & :=\int_{0}^{t} b\left(\tilde{X}_{s}^{\varepsilon}\right) d s+\int_{0}^{t} \int_{\mathbb{R}^{d} \backslash\{0\}} G\left(\tilde{X}_{s}^{\varepsilon}\right)\left(\varphi_{\varepsilon}(s, z)-1\right) z \nu(d z) d s, \\
\bar{M}_{t}^{\varepsilon} & :=\varepsilon \int_{0}^{t} \int_{\mathbb{R}^{d} \backslash\{0\}} G\left(\tilde{X}_{s-}^{\varepsilon}\right) z \tilde{N}^{\frac{\varphi_{\varepsilon}}{\varepsilon}}(d s, d z) .
\end{aligned}
$$

Step 1. We start by showing that the family of processes $\left(J^{\varepsilon}\right)_{\varepsilon \in(0,1]}$ is C-tight (cf. [40-Definition VI.3.25). Let $\rho>0$. For every $\varepsilon>0$ let $(\mathcal{R}(\varepsilon))_{\varepsilon>0}$ be given as in the statement of Proposition 10 , Proposition 10 yields $\varepsilon_{0}>0$ such that $\varepsilon<\varepsilon_{0}$ implies

$$
\overline{\mathbb{P}}\left(\sup _{t \in[0, T]}\left|\tilde{X}_{t}^{\varepsilon}\right|>\mathcal{R}(\varepsilon)\right)<\frac{\rho}{2} .
$$

On the event $\left\{\tilde{\tau}_{\mathcal{R}(\varepsilon)}^{\varepsilon}>T\right\}$ there exists $C_{1}>0$ such that for any $s \leqslant t \leqslant T$ we have

$$
\left|J_{t}^{\varepsilon}-J_{s}^{\varepsilon}\right| \leqslant C_{1}\left(1+\sup _{u \in\left[0, \tilde{\tau}_{\mathcal{R}_{(\varepsilon)}^{\varepsilon}}\right]}\left|\tilde{X}_{u}^{\varepsilon}\right|\right)\left((t-s)+\int_{s}^{t} \int_{\mathbb{R}^{d} \backslash\{0\}}\left|z \| \varphi^{\varepsilon}(u, z)-1\right| \nu(d z) d u\right) .
$$

By Proposition 11 let $\Lambda>0$ and $\varepsilon_{1}>0$ such that $\varepsilon<\varepsilon_{1}$ implies

$$
\overline{\mathbb{E}}\left[\sup _{t \in\left[0, \tilde{\tau}_{\mathcal{R}(\varepsilon)}^{\varepsilon}\right]}\left|\tilde{X}_{t}^{\varepsilon}\right|^{2}\right] \leqslant \Lambda<\infty .
$$

Estimate (26) in Lemma 9 yields some $\delta_{1}>0$ such that for any $s \leqslant t \leqslant T$ with $|t-s| \leqslant \delta_{1}$ it follows

$$
\sup _{g \in \mathfrak{S}^{M}} \int_{s}^{t} \int_{\mathbb{R}^{d} \backslash\{0\}}|z||g(u, z)-1| \nu(d z) d u<\frac{\rho^{2}}{4\left(C_{1}+\Lambda\right)} .
$$

Fix $\delta=\delta_{\rho}<\min \left\{\frac{\rho^{2}}{4\left(C_{1}+\Lambda\right)}, \delta_{1}\right\}$. Due to (33), (34) and (35) $\varepsilon<\varepsilon_{0} \wedge \varepsilon_{1}$ and Markov's inequality yield

$$
\begin{aligned}
\overline{\mathbb{P}}\left(\left|J_{t}^{\varepsilon}-J_{s}^{\varepsilon}\right|>\rho\right) & \leqslant \overline{\mathbb{P}}\left(\left|J_{t}^{\varepsilon}-J_{s}^{\varepsilon}\right|>\rho ; \tilde{\tau}_{\mathcal{R}(\varepsilon)}^{\varepsilon}>T\right)+\overline{\mathbb{P}}\left(\sup _{t \in[0, T]}\left|\tilde{X}_{t}^{\varepsilon}\right|>\mathcal{R}(\varepsilon)\right) \\
& \leqslant \frac{1}{\rho} \overline{\mathbb{E}}\left[\sup _{|t-s|<\delta}\left|J_{t}^{\varepsilon}-J_{s}^{\varepsilon}\right|\right]+\frac{\rho}{2} \leqslant \rho .
\end{aligned}
$$

For $\rho>0$ and $\varepsilon<\varepsilon_{0} \wedge \varepsilon_{1}$ we define the set

$$
\begin{aligned}
K_{\rho}:= & \bigcap_{m \in \mathbb{N}} K_{\rho, m}, \quad \text { where } \\
K_{\rho, m}:= & \left\{f \in C\left([0, T], \mathbb{R}^{d}\right) \mid f(0)=0 \text { and for all } s, t \in[0, T]\right. \\
& \left.|t-s| \leqslant \delta_{\rho 2^{-m}} \text { implies }|f(t)-f(s)|<\rho 2^{-m}\right\} .
\end{aligned}
$$

For every $m \in \mathbb{N}$ the set $K_{\rho, m}$ is a non-empty collection of equicontinuous and uniformly pointwise bounded elements of $C\left([0, T] ; \mathbb{R}^{d}\right)$. Hence due to Arzela-Ascoli's theorem the set $K_{\rho, m}$ is relatively compact in $C\left([0, T] ; \mathbb{R}^{d}\right)$ for every $m \in \mathbb{N}$. Since the non-empty intersection of relatively compact sets in a metric space is relatively compact we conclude that the set $K_{\rho}$ is a non-empty relatively compact set in $C\left([0, T] ; \mathbb{R}^{d}\right)$. Due to (36) it follows that

$$
\overline{\mathbb{P}}\left(J^{\varepsilon} \notin K_{\rho}\right) \leqslant \rho \sum_{m=1}^{\infty} 2^{-m}=\rho,
$$

which implies that $\left(J^{\varepsilon}\right)_{\varepsilon \in(0,1]}$ is $C$-tight. 
Step 2. We show that the family of processes $\left(\bar{M}^{\varepsilon}\right)_{\varepsilon>0}$ is C-tight as $\varepsilon \rightarrow 0$. We fix the scale $(\mathcal{R}(\varepsilon))_{\varepsilon>0}$, the constants $\varepsilon_{0}, C>0$ given in Proposition 10 and

$$
C_{2}:=\sup _{g \in \mathfrak{S}^{M}} \int_{0}^{T} \int_{\mathbb{R}^{d} \backslash\{0\}}|z|^{2} g(s, z) \nu(d z) d s .
$$

Recall that $C_{2}<\infty$ by (24) in Lemma 9 . Hence for every $\kappa>0$ and any $\varepsilon<\varepsilon_{0}$ it follows

$$
\begin{aligned}
& \overline{\mathbb{P}}\left(\left[\bar{M}^{\varepsilon}\right]_{T}>\kappa\right) \leqslant \overline{\mathbb{P}}\left(\left[\bar{M}^{\varepsilon}\right]_{T}>\kappa, \sup _{s \in[0, T]}\left|\tilde{X}_{s}^{\varepsilon}\right| \leqslant \mathcal{R}(\varepsilon)\right)+\overline{\mathbb{P}}\left(\sup _{s \in[0, T]}\left|\tilde{X}_{s}^{\varepsilon}\right|>\mathcal{R}(\varepsilon)\right) \\
& \quad=\overline{\mathbb{P}}\left(\varepsilon^{2} \int_{0}^{T} \int_{\mathbb{R}^{d} \backslash\{0\}}\left|G\left(\tilde{X}_{s-}^{\varepsilon}\right)\right|^{2}|z|^{2} N^{\frac{\varphi_{\varepsilon}}{\varepsilon}}(d s, d z)>\kappa, \sup _{s \in[0, T]}\left|\tilde{X}_{s}^{\varepsilon}\right| \leqslant \mathcal{R}(\varepsilon)\right)+2 e^{-\frac{1}{2} \mathcal{R}(\varepsilon)}+C \varepsilon \mathcal{R}(\varepsilon) \\
& \quad \leqslant \overline{\mathbb{P}}\left(2 L^{2} \varepsilon^{2}\left(1+\mathcal{R}^{2}(\varepsilon)\right) \int_{0}^{T} \int_{\mathbb{R}^{d} \backslash\{0\}}|z|^{2} N^{\frac{\varphi_{\varepsilon}}{\varepsilon}}(d s, d z)>\kappa\right)+2 e^{-\frac{1}{2} \mathcal{R}(\varepsilon)}+C \varepsilon \mathcal{R}(\varepsilon) \\
& \quad \leqslant \frac{2 L^{2} \varepsilon\left(1+\mathcal{R}^{2}(\varepsilon)\right)}{\kappa} \int_{0}^{T} \int_{\mathbb{R}^{d} \backslash\{0\}}|z|^{2} \varphi_{\varepsilon}(s, z) \nu(d z) d s+2 e^{-\frac{1}{2} \mathcal{R}(\varepsilon)}+C \varepsilon \mathcal{R}(\varepsilon) \\
& \quad \leqslant \frac{2 L^{2} C_{2}}{\kappa}\left(1+\mathcal{R}^{2}(\varepsilon)\right) \varepsilon+2 e^{-\frac{1}{2} \mathcal{R}(\varepsilon)}+C \varepsilon \mathcal{R}(\varepsilon) \rightarrow 0, \quad \text { as } \varepsilon \rightarrow 0,
\end{aligned}
$$

since $\varepsilon \mathcal{R}^{2}(\varepsilon) \rightarrow 0$ whenever $\varepsilon \rightarrow 0$. In other words, $\left[\bar{M}^{\varepsilon}\right]_{T} \rightarrow 0$ as $\varepsilon \rightarrow 0$ in probability and therefore in law, which implies that $\left(\left[\bar{M}^{\varepsilon}\right]\right)_{\varepsilon \in(0,1]}$ is $C$-tight.

Step 3. Due to Theorem 6.1.1 in 41 the laws of the family $\tilde{Z}_{t}^{\varepsilon}=x+J_{t}^{\varepsilon}+M_{t}^{\varepsilon}$ are tight in $\mathbb{D}\left([0, T], \mathbb{R}^{d}\right)$. By Prokhorov's Theorem there exists the weak limit of $\left(\tilde{X}^{\varepsilon_{n}}, J^{\varepsilon_{n}}, M^{\varepsilon_{n}}\right)$ for some subsequence $\varepsilon_{n} \rightarrow 0$. Skorokhod's representation's theorem implies that there exists a triplet of random variables $(\tilde{X}, \tilde{\varphi}, 0)$ defined on $(\overline{\mathfrak{M}}, \mathcal{B}(\overline{\mathfrak{M}}), \overline{\mathbb{P}})$ such that $\left(\tilde{X}^{\varepsilon_{n}}, J^{\varepsilon_{n}}, M^{\varepsilon_{n}}\right)$ given by (23) and (32) converges to $(\tilde{X}, \tilde{\varphi}, 0) \overline{\mathbb{P}}$-a.s. as $n \rightarrow \infty$. Due to (25) and the continuity of the functions $b$ and $G$ we can pass to the limit $\tilde{X}_{t}^{\varepsilon_{n}} \rightarrow \tilde{X}_{t}$ pointwise (in $t \in[0, T]$ ) and $\overline{\mathbb{P}}$-a.s. in (23). Hence we have that $\left(\tilde{X}_{s}\right)_{t \in[0, T]}$ satisfies $\overline{\mathbb{P}}$-a.s.

$$
\tilde{X}_{t}=x+\int_{0}^{t} b\left(\tilde{X}_{s}\right) d s+\int_{0}^{t} \int_{\mathbb{R}^{d} \backslash\{0\}} G\left(\tilde{X}_{s}\right)(\tilde{\varphi}(s, z)-1) z \nu(d z) d s, \quad t \in[0, T] .
$$

Therefore we conclude that $\tilde{X}=\mathcal{G}^{0}(\tilde{\varphi})$. Combining that $\varphi$ and $\tilde{\varphi}$ have the same law under $\overline{\mathbb{P}}$ and the C-tightness of $\left(\tilde{X}^{\varepsilon}\right)_{\varepsilon>0}$ implies the $\overline{\mathbb{P}}$-almost sure convergence $\sup _{t \in[0, T]}\left|\tilde{X}_{t}^{\varepsilon_{n}}-\tilde{X}_{t}\right| \rightarrow 0$ as $n \rightarrow \infty$ and hence the convergence in law we infer

$$
\mathcal{G}^{0}(\varphi) \quad \text { is a weak limit point of } \quad \mathcal{G}^{\varepsilon}\left(\varepsilon N^{\frac{1}{\varepsilon} \varphi_{\varepsilon}}\right) .
$$

This finishes the proof.

Proof of Theorem 2. Proposition 12 and 13 imply Condition 2.2(a) and (b) given in [14 for $\left(X^{\varepsilon, x}\right)_{\varepsilon>0}$. Hence Theorem 2.4 of [14] finishes the proof.

\subsection{Some useful consequences}

In the sequel we establish the continuity of the LDP of $\left(X^{\varepsilon, x}\right)_{\varepsilon>0}$ with respect to the initial condition $x \in \mathbb{R}^{d}$.

Proposition 14. Given $T>0$ and $x \in D$ let $F \subset \mathbb{D}\left([0, T], \mathbb{R}^{d}\right)$ be closed and $G \subset \mathbb{D}\left([0, T], \mathbb{R}^{d}\right)$ open with respect to the Skorokhod topology. Then we have

$$
\limsup _{\substack{\varepsilon \rightarrow 0 \\ y \rightarrow x}} \varepsilon \ln \overline{\mathbb{P}}\left(X^{\varepsilon, y} \in F\right) \leqslant-\inf _{f \in F} \mathbb{J}_{x, T}(f),
$$




$$
\liminf _{\substack{\varepsilon \rightarrow 0 \\ y \rightarrow x}} \varepsilon \ln \overline{\mathbb{P}}\left(X^{\varepsilon, y} \in G\right) \geqslant-\inf _{g \in G} \mathbb{J}_{x, T}(g) .
$$

Proof. Due to Theorem 4.4 in [48] the result follows from verifying the following statements.

1. Let $\left(x_{n}\right)_{n \in \mathbb{N}} \subset \mathbb{R}^{d}$ such that $x_{n} \rightarrow x$ as $n \rightarrow \infty$. Given $M>0$ and $\left(g_{n}\right)_{n \in \mathbb{N}} \in \mathfrak{S}^{M}$ such that $\nu_{T}^{g_{n}} \rightarrow \nu_{T}^{g}$ in the vague topology as $n \rightarrow \infty$. Then we obtain

$$
\mathcal{G}^{0, x_{n}}\left(g_{n}\right) \rightarrow \mathcal{G}^{0, x}(g) \text {, as } n \rightarrow \infty .
$$

2. Let $M>0,\left(x_{\varepsilon}\right)_{\varepsilon>0} \subset \mathbb{R}^{d}$ and $\left(\varphi_{\varepsilon}\right)_{\varepsilon>0} \subset \mathfrak{U}_{+}^{M}$ such that $x_{\varepsilon} \rightarrow x$ and $\varphi_{\varepsilon} \Rightarrow \varphi$ in law as $\varepsilon \rightarrow 0$. Then we obtain the following convergence in law

$$
\mathcal{G}^{\varepsilon, x_{\varepsilon}}\left(\varepsilon \tilde{N}^{\frac{1}{\varepsilon} \varphi_{\varepsilon}}\right) \Rightarrow \mathcal{G}^{0, x}(g) \quad \text { as } \varepsilon \rightarrow 0 .
$$

The verification of the conditions above is analogous to the proof of Theorem 2 and we omit its details.

As a consequence of Proposition 14 we derive a uniform LDP for $\left(X^{\varepsilon, x}\right)_{\varepsilon>0}$ when the initial state $x \in K$ for $K \subset D$ a closed (and bounded) set. The proof is virtually the same as the one given in the Brownian case and we omit it. We refer the reader to Corollary 5.6.15 in [22].

Corollary 15. Let $T>0, K \subset D$ be compact, $F \subset \mathbb{D}\left([0, T], \mathbb{R}^{d}\right)$ closed, $G \subset \mathbb{D}\left([0, T], \mathbb{R}^{d}\right)$ open with respect to the $J_{1}$ topology and $x \in D$. Then it follows

$$
\begin{aligned}
& \limsup _{\varepsilon \rightarrow 0} \sup _{y \in K} \varepsilon \ln \overline{\mathbb{P}}\left(X^{\varepsilon, y} \in F\right) \leqslant-\inf _{y \in K, f \in F} \mathbb{J}_{y, T}(f), \\
& \liminf _{\varepsilon \rightarrow 0} \inf _{y \in K} \varepsilon \ln \overline{\mathbb{P}}\left(X^{\varepsilon, y} \in G\right) \geqslant-\inf _{y \in K, g \in G} \mathbb{J}_{y, T}(g) .
\end{aligned}
$$

In the sequel this result is applied to the first exit time problem of $X^{\varepsilon, x}$ from $D$.

\section{The first exit time problem in the small noise limit}

In this section we fix the standing assumptions of the Hypotheses $\mathrm{A}, \mathrm{B}, \mathrm{C}$ and $\mathrm{D}$ for some bounded domain $D \subset \mathbb{R}^{d}, x \in D$ and $\nu \in \mathfrak{M}$.

\subsection{Continuity properties of the cost function}

The following proposition ensures the (local) controllability of the dynamical system given by the controlled integral equation (9) in small balls around the initial position. It plays a crucial role in the proof of the upper bound in Theorem 3 given in the next subsection. We stress that in the more general setting discussed in Subsection 1.4 the following result is stated as Hypothesis $\mathrm{G}$.

Proposition 16. Let Hypotheses $A, B, C$ and $D$ be satisfied. For every $\rho_{0}>0$ there exist a constant $M>0$ and a non-decreasing function $\xi:\left[0, \rho_{0}\right] \rightarrow \mathbb{R}^{+}$with $\lim _{\rho \rightarrow 0} \xi(\rho)=0$ satisfying the following. Then for all $x_{0}, y_{0} \in \mathbb{R}^{d}$ such that $\left|x_{0}-y_{0}\right| \leqslant \rho_{0}$ there exist $\Phi \in C\left(\left[0, \xi\left(\rho_{0}\right)\right], \mathbb{R}^{d}\right)$ and $g \in \mathfrak{S}^{M}$ such that $\Phi\left(\xi\left(\rho_{0}\right)\right)=y_{0}$ and

$$
\Phi(t)=x_{0}+\int_{0}^{t} b(\Phi(s)) d s+\int_{0}^{t} \int_{\mathbb{R}^{d} \backslash\{0\}} G(\Phi(s))(g(s, z)-1) z \nu(d z) d s, \quad t \in\left[0, \xi\left(\rho_{0}\right)\right] .
$$

Proof. For fixed $\rho_{0}>0$ and $x_{0}, y_{0} \in \mathbb{R}^{d}$ such that $\left|x_{0}-y_{0}\right| \leqslant \rho_{0}$ consider the straight line that links $x_{0}$ and $y_{0}$,

$$
\Phi(t):=x_{0}+t \frac{y_{0}-x_{0}}{\rho_{0}}, \quad t \in\left[0, \rho_{0}\right]
$$


Let $\xi(\rho)=\rho, \rho \in\left[0, \rho_{0}\right]$. We observe that $\Phi \in C\left(\left[0, \xi\left(\rho_{0}\right)\right] ; \mathbb{R}^{d}\right)$ and $\Phi\left(\xi\left(\rho_{0}\right)\right)=y_{0}$. The construction of the control function $g \in \mathfrak{S}$ such that (40) holds follows from the next observation. Due to Hypothesis B every vector $x \in \mathbb{R}^{d}$ can be written for some measurable function $f^{x}: \mathbb{R}^{d} \longrightarrow[0, \infty)$ as

$$
x=\int_{\operatorname{supp}(\nu)} z f^{x}(z) \nu(d z) .
$$

For instance we choose the function

$$
f^{x}(z)=\frac{1}{\lambda^{d}\left(B_{R^{\prime}}(x)\right)}\left(\frac{d \nu}{d z}(z)\right)^{-1} \mathbf{1}_{B_{R^{\prime}}(x)}(z)
$$

where $\lambda^{d}$ is the Lebesgue measure on $\left(\mathbb{R}^{d}, \mathcal{B}\left(\mathbb{R}^{d}\right)\right)$ and $R^{\prime}:=\frac{1}{2} d\left(x,(\operatorname{supp}(\nu))^{c}\right) \wedge 1$ with the convention of $d(x, \emptyset)=\infty$. Let $P_{x_{0}, y_{0}}(s)=\frac{y_{0}-x_{0}}{\rho_{0}}-b(\Phi(s))$ for every $s \in\left[0, \xi\left(\rho_{0}\right)\right]$. Set

$$
\begin{gathered}
g:\left[0, \xi\left(\rho_{0}\right)\right] \times \mathbb{R}^{d} \backslash\{0\} \longrightarrow[0, \infty) \\
g(s, z):=1+\frac{\left(\frac{d \nu}{d z}(z)\right)^{-1}}{\lambda^{d}\left(B_{R^{\prime}}\left(P_{x_{0}, y_{0}}(s)\right)\right)} \frac{1}{G(\Phi(s))} \mathbf{1}_{B_{R^{\prime}}\left(P_{x_{0}, y_{0}}(s)\right)} .
\end{gathered}
$$

Since $\nu$ is a finite measure and $g$ is bounded it follows that $g \in \mathfrak{S}$ and (40) holds. This finishes the proof.

We define the following cost function associated to the system (6) which measures the cost of steering $U^{g}$ given in (9) from its initial position $x \in D$ to some point $y \in \mathbb{R}^{d}$ in exactly time $t>0$ by

$$
V(x, y, t):=\inf \left\{\mathcal{E}_{t}(g) \mid g \in \mathfrak{S}_{\varphi} \quad \varphi(s)=U^{g}(s, x), \quad s \in[0, t], \quad \varphi(t)=y\right\} .
$$

The following continuity properties are essentially a consequence of Proposition [16] and are shown in Subsection 4.2 of the appendix.

Lemma 17. Let the assumptions of Theorem $\mathrm{g}$ be satisfied. Then for any $\delta>0$ there exists $\rho>0$ such that

$$
\begin{aligned}
& \sup _{x, y \in B_{\rho}(0)} \inf _{t \in[0,1]} V(x, y, t)<\delta \\
& \sup _{\substack{x, y \in D \\
\inf _{z \in D^{c}}|x-z|+|y-z| \leqslant \rho}} \inf _{t \in[0,1]} V(x, y, t)<\delta .
\end{aligned}
$$

Corollary 18. Let the assumptions of Theorem 3 be satisfied. Then $\bar{V}<\infty$ for $\bar{V}$ being defined by (14).

Proof. We fix $z \in D^{c}$ and take $\rho_{0}=|z|$. By Proposition[16 let $M<\infty, \xi:\left[0, \rho_{0}\right] \longrightarrow \mathbb{R}^{+}$and $g \in \mathfrak{S}^{M}$ such that (40) holds for some $\Phi \in C\left(\left[0, \xi\left(\rho_{0}\right)\right], \mathbb{R}^{d}\right)$ and with $\Phi\left(\xi\left(\rho_{0}\right)\right)=z$. Therefore we have

$$
\bar{V}:=\inf _{z \in D^{c}} V(0, z) \leqslant \int_{0}^{\xi\left(\rho_{0}\right)} \int_{\mathbb{R}^{d} \backslash\{0\}} \ell(g(s, z)) \nu(d z) d s \leqslant M<\infty .
$$

\subsection{Proof of Theorem 3}

Lemma 19. Let $c>0$. There exist $\rho_{0}>0$ and $s_{0}>0$ such that for any $\rho \in\left(0, \rho_{0}\right]$ it holds the limit

$$
\liminf _{\varepsilon \rightarrow 0} \varepsilon \ln \inf _{x \in B_{\rho}(0)} \overline{\mathbb{P}}\left(\sigma^{\varepsilon}(x) \leqslant s_{0}\right)>-(\bar{V}+c),
$$

where the potential height $\bar{V}$ is given in equation (14). 
Proof. Let $\rho_{0}>0$ be small enough such that the inequalities of (41) and (42) in Lemma 17 are satisfied for $\delta=\frac{c}{2}$ and $\rho \leqslant \rho_{0}$. Hence we may choose $x \in B_{\rho}(0)$ and a path $\varphi_{1}^{x} \in C\left(\left[0, s_{x}\right], \mathbb{R}^{d}\right)$ satisfying $\varphi_{1}^{x}(0)=x, \varphi_{1}^{x}\left(s_{x}\right)=0$ such that

$$
\mathbb{J}_{x, s_{x}}\left(\varphi_{1}^{x}\right) \leqslant \frac{c}{2} .
$$

With the help of [42) in Lemma 17 and Proposition [16 we may choose $z \in D^{c} \cap \operatorname{supp}(\nu), s_{z}>0$, $\varphi_{2}^{z} \in C\left(\left[0, s_{z}\right], \mathbb{R}^{d}\right)$ such that $\varphi_{2}^{z}(0)=0, \varphi_{2}^{z}\left(s_{z}\right)=z$ and

$$
\mathbb{J}_{0, s_{z}}\left(\varphi_{2}^{z}\right) \leqslant \bar{V}+\frac{c}{2} .
$$

Let $\varphi_{3}$ be the solution of the differential equation $\dot{\varphi}_{3}=b\left(\varphi_{3}\right)$ with $\varphi_{3}(0)=z$. We set $s_{0}=s_{x}+s_{z}+\delta^{\prime}$ with $\delta^{\prime}>0$ such that $\varphi_{3}\left(\left[0, \delta^{\prime}\right]\right) \subset D^{c}$ and define

$$
\Phi^{x}(t):= \begin{cases}\varphi_{1}^{x}(t) & \text { if } t \in\left[0, s_{x}\right], \\ \varphi_{2}^{z}\left(t-s_{x}\right) & \text { if } t \in\left(s_{x}, s_{z}+s_{x}\right], \\ \varphi_{3}\left(t-s_{z}-s_{x}\right) & \text { if } t \in\left(s_{x}+s_{z}, s_{0}\right] .\end{cases}
$$

Then the concatenation of the paths yields

$$
\mathbb{J}_{x, s_{0}}\left(\Phi^{x}\right) \leqslant \mathbb{J}_{x, s_{x}}\left(\varphi_{1}^{x}\right)+\mathbb{J}_{0, s_{z}}\left(\varphi_{2}^{z}\right)+0 \leqslant \bar{V}+c .
$$

Let $\Delta=d(z, \bar{D})$ and consider the open set

$$
\mathcal{O}=\bigcup_{x \in B_{\rho_{0}}(0)}\left\{\psi \in \mathbb{D}\left(\left[0, s_{0}\right], \mathbb{R}^{d}\right) \mid d_{J_{1}}\left(\psi, \Phi^{x}\right)<\frac{\Delta}{2}\right\}
$$

The constructed path $\Phi^{x}$ visits $z$ by definition and stays outside of $D$ in the time interval $\left[s_{x}+s_{z}, s_{0}\right]$, due to the choice of $z \in D^{c}$ and the continuity of $\varphi_{3}$. By definition of $\mathcal{O}$ every path $\psi \in \mathcal{O}$ exits $D$ before time $s_{0}$. We show this claim by contradiction. Fix $\psi \in \mathcal{O}$. Let us suppose that $\psi\left(\left[0, s_{0}\right]\right) \subset D$. This implies that

$$
d\left(z, \overline{\psi\left(\left[0, s_{0}\right]\right)}\right)>\Delta .
$$

Since $\psi \in \mathcal{O}$ we have $d_{J_{1}}\left(\psi, \Phi^{x}\right)<\frac{\Delta}{2}$, that is, there is an increasing homeomorphism $\lambda:\left[0, s_{0}\right] \rightarrow\left[0, s_{0}\right]$ such that

$$
\sup _{t \in\left[0, s_{0}\right]}\left|\psi(\lambda(t))-\Phi^{x}(t)\right|<\frac{\Delta}{2}
$$

In particular,

$$
\left|\psi\left(\lambda\left(s_{z}+s_{x}\right)\right)-\Phi^{x}\left(s_{z}+s_{x}\right)\right|=\left|\psi\left(\lambda\left(s_{z}+s_{x}\right)\right)-z\right|<\frac{\Delta}{2},
$$

which contradicts (43). Corollary 15 yields

$$
\begin{aligned}
\liminf _{\varepsilon \rightarrow 0} \varepsilon \ln \inf _{x \in B_{\rho_{0}}(0)} \overline{\mathbb{P}}\left(\sigma^{\varepsilon}(x) \leqslant s_{0}\right) & \geqslant \liminf _{\varepsilon \rightarrow 0} \varepsilon \ln \inf _{x \in B_{\rho_{0}}(0)} \overline{\mathbb{P}}\left(X^{\varepsilon, x} \in \mathcal{O}\right) \\
& \geqslant-\sup _{x \in B_{\rho_{0}}(0)} \inf _{\psi \in \mathcal{O}} \mathbb{J}_{x, s_{0}}(\psi) \geqslant-\sup _{x \in B_{\rho_{0}}(0)} \mathbb{J}_{x, s_{0}}\left(\Phi^{x}\right) \geqslant-(\bar{V}+c),
\end{aligned}
$$

which finishes the proof.

For fixed $x \in D$ and small $\varepsilon>0$ we show that the probability of $X^{\varepsilon, x}$ staying in $D$ in the long run without hitting a small neighborhood of 0 is exponentially negligible. For given $\rho>0$ such that $\bar{B}_{\rho}(0) \subset D$, we define

$$
\vartheta_{\rho}^{\varepsilon}(x):=\inf \left\{t \geqslant 0|| X_{t}^{\varepsilon, x} \mid \leqslant \rho \text { or } X_{t}^{\varepsilon, x} \in D^{c}\right\} .
$$


Lemma 20. We have

$$
\lim _{t \rightarrow \infty} \limsup _{\varepsilon \rightarrow 0} \varepsilon \ln \sup _{x \in D} \overline{\mathbb{P}}\left(\vartheta_{\rho}^{\varepsilon}(x)>t\right)=-\infty .
$$

Proof. Let us fix $\rho>0$. For $t \geqslant 0$ we define the subsets of $\mathbb{D}\left([0, t], \mathbb{R}^{d}\right)$

$$
\begin{aligned}
\mathcal{G}_{t}:= & \left\{\Phi \in \mathbb{D}\left([0, t], \mathbb{R}^{d}\right) \mid \Phi(s) \in \overline{D \backslash B_{\rho}(0)} \text { for all } s \in[0, t]\right\} \text { and } \\
\tilde{\mathcal{G}}_{t}:=\left\{\Phi \in \mathbb{D}\left([0, t], \mathbb{R}^{d}\right) \mid \Phi(s) \in \overline{D \backslash B_{\rho}(0)} \text { for all } s \in[0, t]\right. & \{\text { except in a countable number of points }\} .
\end{aligned}
$$

In Lemma25in Subsection 4.3 of the appendix it is shown that due to right continuity we have $\tilde{\mathcal{G}}_{t}=\mathcal{G}_{t}$ and $\tilde{\mathcal{G}}_{t}$ is a closed set in $\mathbb{D}\left([0, t], \mathbb{R}^{d}\right)$ with respect to the Skorokhod topology. By the definition of $\mathcal{G}_{t}$ and Corollary 15 we have

$$
\begin{aligned}
\limsup _{\varepsilon \rightarrow 0} \varepsilon \ln \sup _{x \in D} \overline{\mathbb{P}}\left(\vartheta_{\rho}^{\varepsilon}(x)>t\right) & \leqslant \limsup _{\varepsilon \rightarrow 0} \ln \sup _{x \in \overline{D \backslash B_{\rho}(0)}} \overline{\mathbb{P}}\left(\vartheta_{\rho}^{\varepsilon}(x)>t\right) \leqslant-\inf _{x \in \overline{D \backslash B_{\rho}(0)}} \inf _{\psi \in \tilde{\mathcal{G}}_{t}} \mathbb{J}_{x, t}(\psi) \\
& =-\inf _{x \in D \backslash B_{\rho}(0)} \inf _{\psi \in \mathcal{G}_{t}} \mathbb{J}_{x, t}(\psi)=-\inf _{\psi \in \mathcal{G}_{t}} \mathbb{J}_{\psi(0), t}(\psi) .
\end{aligned}
$$

Claim: We have

$$
\lim _{t \rightarrow \infty} \inf _{\psi \in \mathcal{G}_{t}} \mathbb{J}_{\psi(0), t}(\psi)=\infty .
$$

Let $\left(\varphi_{t}\right)_{t \geqslant 0}$ be the dynamical system associated to $\dot{\varphi}_{t}=b\left(\varphi_{t}\right)$ on $\mathbb{R}^{d}$. Due to Hypothesis $\mathrm{A}$ for any $x \in D \backslash \bar{B}_{\rho}(0)$ there exists $t_{x} \geqslant 0$ such that $\varphi\left(t_{x}\right) \in B_{\frac{\rho}{2}}(0)$. We define the open neighborhood $O_{x}:=\varphi^{-1}\left(B_{\frac{\rho}{2}}(0)\right)$ of $x$ in $\mathbb{R}^{d}$. By compactness there are $k \in \mathbb{N}$ and $x_{1}, \ldots, x_{k} \in D \backslash \bar{B}_{\rho}(0)$ such that $\bigcup_{i=1}^{k} O_{x_{i}} \supset\left(D \backslash \bar{B}_{\rho}(0)\right)$. We set $s=t_{x_{1}} \vee \cdots \vee t_{x_{k}}$. Before time $s$ any path that solves $\dot{\varphi}_{t}=b\left(\varphi_{t}\right)$, with initial condition in $D \backslash \bar{B}_{\rho}(0)$ hits $B_{\frac{\rho}{2}}(0)$. We argue by contradiction. Assume that

$$
\lim _{t \rightarrow+\infty} \inf _{\psi \in \mathcal{G}_{t}} \mathbb{J}_{\psi(0), t}(\psi)<\infty .
$$

Let us fix $M>0$ such that for any $n \in \mathbb{N}$ there exists $\psi^{n} \in \mathcal{G}_{n s}$ verifying $\mathbb{J}_{\psi^{n}(0), n s}\left(\psi^{n}\right) \leqslant M$. For $k=0, \ldots, n-1$ let

$$
\psi^{n, k}(t):=\psi^{n}(k \cdot(s-t)), t \in[0, s] .
$$

Hence $\psi_{n, k} \in \mathcal{G}_{s}$ and

$$
M \geqslant \mathbb{J}_{\psi^{n}(0), n s}\left(\psi^{n}\right)=\sum_{i=0}^{n-1} \mathbb{J}_{\psi^{n}(k s), s}\left(\psi^{n, k}\right) \geqslant n \min _{0 \leqslant k \leqslant n-1} \mathbb{J}_{\psi^{n, k}(0), s}\left(\psi^{n, k}\right) .
$$

We finally show the existence of a sequence $\left(\psi^{n}\right)_{n \in \mathbb{N}}$ in $\mathcal{G}_{t}$ such that

$$
\lim _{n \rightarrow \infty} \mathbb{J}_{\psi^{n}(0), s}\left(\psi^{n}\right)=0 .
$$

First we see that the set

$$
\left\{\psi \in \mathbb{D}\left([0, s], \mathbb{R}^{d}\right) \mid \psi(0) \in \overline{D \backslash B_{\rho}(0)}, \mathbb{J}_{\psi(0), s}(\psi) \leqslant 1\right\}
$$

is a closed subset of the compact set $\left\{\psi \in \mathbb{D}\left([0, s], \mathbb{R}^{d}\right) \mid \mathbb{J}_{\psi(0), s}(\psi) \leqslant 1\right\}$. The compactness comes from the fact that $\mathbb{J}_{\psi(0), s}$ is a good rate function with respect to the Skorokhod topology. Hence the sequence $\left(\psi^{n}\right)_{n \in \mathbb{N}}$ has a limit point in $\mathcal{G}_{s}$ which we call $\bar{\psi}$. Since $\mathbb{J}_{\psi(0), s}=\inf _{x \in \mathbb{R}^{d}} \mathbb{J}_{x, s}$ is lower semicontinuous and due to (49) it follows that $\mathbb{J}_{\bar{\psi}(0), s}(\bar{\psi})=0$. Due to the definition of rate function in (11), the structure of the controlled paths in (9) and (7) this implies that $\bar{\psi}$ solves $\dot{\bar{\psi}}_{t}=b\left(\bar{\psi}_{t}\right)$ with $\bar{\psi}(0) \in D \backslash \bar{B}_{\rho}(0)$. Therefore $\bar{\psi}$ reaches $B_{\frac{\rho}{2}}(0)$ before time $s$, which contradicts $\bar{\psi} \in \mathcal{G}_{s}$ and thus assumption (48). Combining inequality (46) and (47) yields the desired result (45). 
Theorem 5. For $x \in D$ and $\delta>0$ we have

$$
\begin{aligned}
& \liminf _{\varepsilon \rightarrow 0} \varepsilon \ln \overline{\mathbb{P}}\left(\sigma^{\varepsilon}(x)<e^{\frac{\bar{v}+\delta}{\varepsilon}}\right)=1 \text { and } \\
& \limsup _{\varepsilon \rightarrow 0} \varepsilon \ln \overline{\mathbb{E}}\left[\sigma^{\varepsilon}(x)\right] \leqslant \bar{V}+\frac{\delta}{2} .
\end{aligned}
$$

Proof. The proof consists of two steps.

Claim 1. For any $\delta>0$ there are $T>0, c>0$ and $\varepsilon_{0} \in(0,1]$ such that $\varepsilon \in\left(0, \varepsilon_{0}\right]$ implies

$$
\inf _{x \in D} \overline{\mathbb{P}}\left(\sigma^{\varepsilon}(x) \leqslant T\right) \geqslant c e^{-\frac{\bar{v}+\frac{\delta}{2}}{\varepsilon}} .
$$

We first observe that by Lemma 19 for every $\delta>0$ there are $t_{0}>0$ and $\rho>0$ such that

$$
\liminf _{\varepsilon \rightarrow 0} \varepsilon \ln \inf _{x \in B_{\rho}(0)} \overline{\mathbb{P}}\left(\sigma^{\varepsilon}(x) \leqslant t_{0}\right)>-\left(\bar{V}+\frac{\delta}{4}\right) .
$$

For the fixed value $\rho>0$ and any $r>0$ Lemma 20 yields $t_{1}>0$ and $\varepsilon_{0} \in(0,1]$ such that $\varepsilon<\varepsilon_{0}$ implies

$$
\varepsilon \ln \sup _{x \in D} \overline{\mathbb{P}}\left(\vartheta_{\rho}^{\varepsilon}(x)>t_{1}\right)<-r .
$$

In addition, let $\tilde{c}>0$ and $\varepsilon_{0} \in(0,1]$ sufficiently small such that for $\varepsilon \in\left(0, \varepsilon_{0}\right]$ it follows $1-e^{-\frac{r}{\varepsilon}}>\tilde{c} e^{-\frac{\delta}{4 \varepsilon}}$. Since $\left\{\vartheta_{\rho}^{\varepsilon}(x)<\sigma^{\varepsilon}(x)\right\}=\left\{X_{\vartheta_{\rho}^{\varepsilon}(x)}^{\varepsilon, x} \in \bar{B}_{\rho}(0)\right\}$ we have on this event

$$
\sigma^{\varepsilon}(x)=\vartheta_{\rho}^{\varepsilon}(x)+\sigma^{\varepsilon}\left(X_{\vartheta_{\rho}^{\varepsilon}(x)}^{\varepsilon, x}\right) \circ \Theta_{\vartheta_{\rho}^{\varepsilon}(x)},
$$

where $\Theta_{s}$ is the canonical shift by time $s$ on the path space $\mathbb{D}\left([0, \infty), \mathbb{R}^{d}\right)$. Using the homogeneous strong Markov property of $X^{\varepsilon, x}$ we obtain for any fixed $\varepsilon \in\left(0, \varepsilon_{0}\right]$ and $x \in D$

$$
\begin{aligned}
\overline{\mathbb{P}}\left(\sigma^{\varepsilon}(x) \leqslant t_{0}+t_{1}\right) & \geqslant \overline{\mathbb{P}}\left(\vartheta_{\rho}^{\varepsilon}(x) \leqslant t_{1} \text { and } \sigma^{\varepsilon}\left(X_{\vartheta_{\rho}^{\varepsilon}(x)}^{\varepsilon, x}\right) \leqslant t_{0}\right) \\
& =\overline{\mathbb{P}}\left(\vartheta_{\rho}^{\varepsilon}(x) \leqslant t_{1}\right) \overline{\mathbb{P}}\left(\sigma^{\varepsilon}\left(X_{\vartheta_{\rho}^{\varepsilon}(x)}^{\varepsilon, x}\right) \leqslant t_{0} \mid \vartheta_{\rho}^{\varepsilon}(x) \leqslant t_{1}\right) \\
& \geqslant \inf _{y \in D} \overline{\mathbb{P}}\left(\vartheta_{\rho}^{\varepsilon}(y) \leqslant t_{1}\right) \inf _{x \in B_{\rho}(0)} \overline{\mathbb{P}}\left(\sigma^{\varepsilon}(x) \leqslant t_{0}\right) \\
& \geqslant c e^{-\frac{\bar{V}+\frac{\delta}{4}}{\varepsilon}} \tilde{c} e^{-\frac{\delta}{4 \varepsilon}} \geqslant c \tilde{c} e^{-\frac{\bar{V}+\frac{\delta}{4}}{\varepsilon}}\left(1-e^{-\frac{r}{\varepsilon}}\right)=c \tilde{c} e^{-\frac{\bar{V}+\frac{\delta}{2}}{\varepsilon}} .
\end{aligned}
$$

Setting $T=t_{0}+t_{1}$ and renaming the constants we finish the proof of Claim 1 ,

Step 2: We continue with the proof of the limit (50) and set $q^{\varepsilon}:=\inf _{x \in D} \overline{\mathbb{P}}\left(\sigma^{\varepsilon}(x) \leqslant T\right)$ for the time $T>0$ given in Claim 1 Claim 1 yields $q^{\varepsilon}>0$ for all $\varepsilon \in\left(0, \varepsilon_{0}\right]$. For any $k \in \mathbb{N}$ and $x \in D$ we consider the family of events $\left\{\sigma^{\varepsilon}(x)>k T\right\}$ for which we derive the following recursion

$$
\begin{aligned}
\overline{\mathbb{P}}\left(\sigma^{\varepsilon}(x)>(k+1) T\right) & =\left(1-\overline{\mathbb{P}}\left(\sigma^{\varepsilon}(x) \leqslant(k+1) T \mid \sigma^{\varepsilon}(x)>k T\right)\right) \cdot \overline{\mathbb{P}}\left(\sigma^{\varepsilon}(x)>k T\right) \\
& \leqslant\left(1-q^{\varepsilon}\right) \cdot \overline{\mathbb{P}}\left(\sigma^{\varepsilon}(x)>k T\right), \quad k \in \mathbb{N} .
\end{aligned}
$$

Solving the recursion above in $k \in \mathbb{N}$ we obtain for any $\varepsilon \in\left(0, \varepsilon_{0}\right]$

$$
\sup _{x \in D} \overline{\mathbb{P}}\left(\sigma^{\varepsilon}(x)>k T\right) \leqslant\left(1-q^{\varepsilon}\right)^{k}, \quad k \in \mathbb{N} .
$$

This implies the following bound

$$
\sup _{x \in D} \overline{\mathbb{E}}\left[\sigma^{\varepsilon}(x)\right]=\sup _{x \in D} T \int_{0}^{\infty} \overline{\mathbb{P}}\left(\sigma^{\varepsilon}(x)>T s\right) d s \leqslant T \sup _{x \in D} \sum_{k=0}^{\infty} \overline{\mathbb{P}}\left(\sigma^{\varepsilon}(x)>k T\right) \leqslant T \sum_{k=0}^{\infty}\left(1-q^{\varepsilon}\right)^{k}=\frac{T}{q^{\varepsilon}} .
$$


Since we have $q^{\varepsilon} \geqslant e^{-\frac{\bar{V}+\frac{\delta}{2}}{\varepsilon}}$ for $\varepsilon \in\left(0, \varepsilon_{0}\right]$ we obtain

$$
\sup _{x \in D} \overline{\mathbb{E}}\left[\sigma^{\varepsilon}(x)\right] \leqslant T e^{\frac{\bar{v}+\frac{\delta}{2}}{\varepsilon}} .
$$

Chebyshev's inequality implies for all $x \in D$ and $\varepsilon \in\left(0, \varepsilon_{0}\right]$,

$$
\overline{\mathbb{P}}\left(\sigma^{\varepsilon}(x) \geqslant e^{\frac{\bar{V}+\delta}{\varepsilon}}\right) \leqslant e^{-\frac{\bar{V}+\delta}{\varepsilon}} \overline{\mathbb{E}}\left[\sigma^{\varepsilon}(x)\right] \leqslant e^{-\frac{\delta}{2 \varepsilon}} .
$$

Sending $\varepsilon \rightarrow 0$ we conclude.

Lemma 21. For any $x \in D$ and $\rho>0$ such that $\bar{B}_{\rho}(0) \subset D$ we have

$$
\lim _{\varepsilon \rightarrow 0} \overline{\mathbb{P}}\left(X_{\vartheta_{\rho}^{\varepsilon}(x)}^{\varepsilon, x} \in \bar{B}_{\rho}(0)\right)=1 .
$$

Proof. We fix $\rho>0$ and $x \in D \backslash \bar{B}_{\rho}(0)$. Otherwise the result is trivial. Due to Hypothesis $\mathrm{A}, 1$ there exists $T>0$ such that $X_{t}^{0, x} \in B_{\frac{\rho}{2}}$ for all $t \geqslant T$. Hypothesis $\mathrm{D}$ yields

$$
\Delta:=\rho \wedge \operatorname{dist}\left(\left\{X_{t}^{0, x} \mid t \in[0, T]\right\}, D^{c}\right)>0 .
$$

Hence it follows that

$$
\begin{aligned}
\left\{X_{\vartheta_{\rho}^{\varepsilon}(x)}^{\varepsilon, x} \in D^{c}\right\} & \subset\left\{\sup _{t \in\left[0, T \wedge \vartheta_{\rho}^{\varepsilon}(x)\right]}\left|X_{t}^{\varepsilon, x}-X_{t}^{0, x}\right|>\frac{\Delta}{2}\right\} \\
& \subset\left\{\sup _{t \in\left[0, T \wedge \vartheta_{\rho}^{\varepsilon}(x)\right]}\left|X_{t}^{\varepsilon, x}-\overline{\mathbb{E}}\left[X_{t}^{\varepsilon, x}\right]\right|>\frac{\Delta}{4}\right\} \cup\left\{\sup _{t \in\left[0, T \wedge \vartheta_{\rho}^{\varepsilon}(x)\right]}\left|\overline{\mathbb{E}}\left[X_{t}^{\varepsilon, x}\right]-X_{t}^{0, x}\right|>\frac{\Delta}{4}\right\} .
\end{aligned}
$$

We first show that for $\varepsilon$ sufficiently small the second event is empty. Indeed,

$$
\left|\overline{\mathbb{E}}\left[X_{t}^{\varepsilon, x}\right]-X_{t}^{0, x}\right|^{2}=\left|\overline{\mathbb{E}}\left[X_{t}^{\varepsilon, x}-X_{t}^{0, x}\right]\right|^{2} \leqslant \overline{\mathbb{E}}\left[\left|X_{t}^{\varepsilon, x}-X_{t}^{0, x}\right|^{2}\right] \text { for all } t \geqslant 0,
$$

and by Itô's formula we have for $t \leqslant T$

$$
\begin{aligned}
\overline{\mathbb{E}}\left[\left|X_{t \wedge \vartheta_{\rho}^{\varepsilon}(x)}^{\varepsilon, x}-X_{\wedge \vartheta_{\rho}^{\varepsilon}(x)}^{0, x}\right|^{2}\right]= & 2 \overline{\mathbb{E}}\left[\int_{0}^{\wedge \vartheta_{\rho}^{\varepsilon}(x)}\left\langle X_{s}^{\varepsilon, x}-X_{s}^{0, x}, b\left(X_{s}^{\varepsilon, x}\right)-b\left(X_{s}^{0, x}\right)\right\rangle d s\right] \\
& +\overline{\mathbb{E}}\left[\int_{0}^{t \wedge \vartheta_{\rho}^{\varepsilon}(x)}\left|\varepsilon G\left(X_{s-}^{\varepsilon, x}, z\right)\right|^{2} \frac{1}{\varepsilon} \nu(d s) d s\right] \\
\leqslant & \left.-2 c_{1} \overline{\mathbb{E}} \int_{0}^{t \wedge \vartheta_{\rho}^{\varepsilon}(x)}\left|X_{s}^{\varepsilon, x}-X_{s}^{0, x}\right|^{2} d s\right] \\
& \left.+\varepsilon \overline{\mathbb{E}}\left[\left.\int_{0}^{t \wedge \vartheta_{\rho}^{\varepsilon}(x)} \int_{\mathbb{R}^{d}} L\left(1+\mid X_{s-}^{\varepsilon, x}, z\right)\right|^{2}\right) \nu(d z) d s\right] .
\end{aligned}
$$

We the estimate

$$
\overline{\mathbb{E}}\left[\left|X_{t \wedge \vartheta_{\rho}^{\varepsilon}(x)}^{\varepsilon, x}-X_{t \wedge \vartheta_{\rho}^{\varepsilon}(x)}^{0, x}\right|^{2}\right] \leqslant \varepsilon T L\left(1+\operatorname{diam}(D)^{2}\right) \int_{\mathbb{R}^{d}}|z|^{2} \nu(d z)
$$

and hence for all $\varepsilon<\frac{\Delta}{4} C, C=\left(T L\left(1+\operatorname{diam}(D)^{2}\right) \int_{\mathbb{R}^{d}}|z|^{2} \nu(d z)\right)^{-1}$ we have

$$
\left|\overline{\mathbb{E}}\left[X_{t}^{\varepsilon, x}\right]-x\right| \leqslant \frac{\Delta}{4} .
$$

Therefore it follows for any $\lambda>0$ that

$$
\overline{\mathbb{P}}\left(X_{\vartheta_{\rho}^{\varepsilon}(x)}^{\varepsilon, x} \in D^{c}\right) \leqslant \overline{\mathbb{P}}\left(\sup _{t \in\left[0, T \wedge \vartheta_{\rho}^{\varepsilon}(x)\right]}\left|X_{t}^{\varepsilon, x}-\overline{\mathbb{E}}\left[X_{t}^{\varepsilon, x}\right]\right|>\frac{\Delta}{4}\right)
$$




$$
\begin{aligned}
& \leqslant \overline{\mathbb{P}}\left(\sup _{t \in\left[0, T \wedge \vartheta_{\rho}^{\varepsilon}(x)\right]}\left|X_{t}^{\varepsilon, x}-\overline{\mathbb{E}}\left[X_{t}^{\varepsilon, x}\right]\right|>\frac{\Delta}{4} \mid\left[X^{\varepsilon, x}-\overline{\mathbb{E}}\left[X^{\varepsilon, x}\right]\right]_{T \wedge \vartheta_{\rho}^{\varepsilon}(x)} \leqslant \lambda\right) \\
& \quad+\overline{\mathbb{P}}\left(\left[X^{\varepsilon, x}-\overline{\mathbb{E}}\left[X^{\varepsilon, x}\right]\right]_{T \wedge \vartheta_{\rho}^{\varepsilon}(x)}>\lambda\right) .
\end{aligned}
$$

In this case the Bernstein-type inequality for local martingales given by Theorem 3.3 of [25] reads for $X_{t}^{\varepsilon, x}-\overline{\mathbb{E}}\left[X_{t}^{\varepsilon, x}\right]$ as follows

$$
\overline{\mathbb{P}}\left(\sup _{t \in\left[0, T \wedge \vartheta_{\rho}^{\varepsilon}(x)\right]}\left|X_{t}^{\varepsilon, x}-\overline{\mathbb{E}}\left[X_{t}^{\varepsilon, x}\right]\right|>\frac{\rho}{2} \mid\left[X^{\varepsilon, x}-\overline{\mathbb{E}}\left[X^{\varepsilon, x}\right]\right]_{T \wedge \vartheta_{\rho}^{\varepsilon}(x)} \leqslant \lambda\right) \leqslant 2 \exp \left(-\frac{1}{2} \frac{\rho^{2}}{4 \lambda}\right) .
$$

Hypotheses $\mathrm{C}$ and $\mathrm{D}$ yield some constant $C>0$ such that for $\varepsilon>0$ small enough

$$
\left[X^{\varepsilon, x}-\overline{\mathbb{E}}\left[X^{\varepsilon, x}\right]\right]_{T \wedge \vartheta_{\rho}^{\varepsilon}(x)} \leqslant C \varepsilon^{2} \int_{0}^{T} \int_{\mathbb{R}^{d} \backslash\{0\}}|z|^{2} N^{\frac{1}{\varepsilon}}(d s, d z) .
$$

Therefore we obtain for $\varepsilon>0$ small enough

$$
\overline{\mathbb{P}}\left(\left[X^{\varepsilon, x}-\overline{\mathbb{E}}\left[X^{\varepsilon, x}\right]\right]_{T \wedge \vartheta_{\rho}^{\varepsilon}(x)}>\lambda\right) \leqslant \frac{\varepsilon^{2} C}{\lambda} \overline{\mathbb{E}}\left[\int_{0}^{T} \int_{\mathbb{R}^{d} \backslash\{0\}}|z|^{2} N^{\frac{1}{\varepsilon}}(d s, d z)\right] \leqslant C C_{\nu}^{2} T \frac{\varepsilon}{\lambda}
$$

where $C_{\nu}^{2}:=\int_{\mathbb{R}^{d} \backslash\{0\}}|z|^{2} \nu(d z)<\infty$ due to the fact that $\nu$ is a Lévy measure respecting the integrability condition (5). Hence choosing $\lambda:=\lambda_{\varepsilon}:=\varepsilon^{\frac{1}{2}}$ the inequalities (53) and (54) imply with (52) that

$$
\overline{\mathbb{P}}\left(X_{\vartheta_{\rho}^{\varepsilon}(x)}^{\varepsilon, x} \notin D\right) \leqslant 2 \exp \left(-\frac{1}{2} \frac{\Delta^{2}}{4 \sqrt{\varepsilon}}\right)+C T c_{\nu}^{2} \varepsilon^{\frac{1}{2}} .
$$

Sending $\varepsilon \rightarrow 0$ we infer the desired result.

Lemma 22. For any $\rho>0$ and $c>0$ we have

$$
\limsup _{\varepsilon \rightarrow 0} \varepsilon \ln \sup _{x \in D} \overline{\mathbb{P}}\left(\sup _{t \in\left[0, \varepsilon \wedge \sigma^{\varepsilon}(x)\right]}\left|X_{t}^{\varepsilon, x}-x\right| \geqslant \rho\right)<-c .
$$

Proof. We fix $\rho>0$ and $x \in D \backslash \bar{B}_{\rho}(0)$. Otherwise the result is trivial. Let $p, q>0$ be the constants appearing in Hypothesis E.3. First note that due to the Hypothesis E.2 there is a constant $C>0$ such that for any scale $\theta_{\varepsilon} / \varepsilon \rightarrow \infty$ as $\varepsilon \rightarrow 0$ we have

$$
\begin{aligned}
\overline{\mathbb{P}}\left(\inf \left\{t>0|| \varepsilon \Delta_{t} L^{\varepsilon} \mid>\theta_{\varepsilon}\right\} \leqslant \varepsilon\right) & =1-\exp \left(-\varepsilon(1 / \varepsilon) \nu\left(\left(\theta_{\varepsilon} / \varepsilon\right) B_{1}^{c}(0)\right)\right) \\
& \leqslant 1-\exp \left(-C e^{-\Gamma \frac{\theta_{\varepsilon}^{2}}{\varepsilon^{2}}}\right) \leqslant 2 C e^{-\Gamma \frac{\theta_{\varepsilon}^{2}}{\varepsilon^{2}}} .
\end{aligned}
$$

For the threshold $\theta_{\varepsilon}=\sqrt{\varepsilon}|\ln (\varepsilon)|^{q}$ this limit is asymptotically exponentially negligible as $\varepsilon \rightarrow 0$. Hence we consider $X^{\varepsilon, x}$ to be conditioned to have jumps with size less or equal to $\sqrt{\varepsilon}|\ln (\varepsilon)|^{q}$. We denote this process by $\mathcal{X}^{\varepsilon, x}$. In addition, we have

$$
\left\{\sup _{t \in\left[0, \varepsilon \wedge \sigma^{\varepsilon}(x)\right]}\left|\mathcal{X}_{t}^{\varepsilon, x}-x\right| \geqslant \rho\right\} \subset\left\{\sup _{t \in\left[0, \varepsilon \wedge \sigma^{\varepsilon}(x)\right]}\left|\mathcal{X}_{t}^{\varepsilon, x}-\overline{\mathbb{E}}\left[\mathcal{X}_{t}^{\varepsilon, x}\right]\right| \geqslant \frac{\rho}{2}\right\} \cup\left\{\sup _{t \in[0, \varepsilon]}\left|\overline{\mathbb{E}}\left[\mathcal{X}_{t}^{\varepsilon, x}\right]-x\right|>\frac{\rho}{2}\right\} .
$$

We first show that for $\varepsilon$ sufficiently small the second event is empty. For any $t>0$ we have

$$
\left|\overline{\mathbb{E}}\left[\mathcal{X}_{t}^{\varepsilon, x}\right]-x\right|^{2}=\left|\overline{\mathbb{E}}\left[\mathcal{X}_{t}^{\varepsilon, x}-x\right]\right|^{2} \leqslant \overline{\mathbb{E}}\left[\left|\mathcal{X}_{t}^{\varepsilon, x}-x\right|^{2}\right] .
$$

We continue with the help of $b(0)=0$ and Itô's formula

$$
\left.\overline{\mathbb{E}}\left[\left|\mathcal{X}_{t}^{\varepsilon, x}-x\right|^{2}\right]=2 \overline{\mathbb{E}} \int_{0}^{t}\left\langle\mathcal{X}_{s}^{\varepsilon, x}, b\left(X_{s}^{\varepsilon, x}\right)\right)\right\rangle d s+\overline{\mathbb{E}} \int_{0}^{t} \int_{|z| \leqslant \sqrt{\varepsilon}|\ln (\varepsilon)|^{q}}\left|\varepsilon G\left(\mathcal{X}_{s-}^{\varepsilon, x}, z\right)\right|^{2} \frac{1}{\varepsilon} \nu(d s) d s
$$




$$
\begin{aligned}
& \leqslant-2 c_{1} \overline{\mathbb{E}} \int_{0}^{t}\left|\mathcal{X}_{s}^{\varepsilon, x}\right|^{2} d s+\varepsilon \int_{0}^{t} \int_{|z| \leqslant \sqrt{\varepsilon}|\ln (\varepsilon)|^{q}} L\left(1+\overline{\mathbb{E}}\left[\left|\mathcal{X}_{s-}^{\varepsilon, x}\right|^{2}\right]\right)|z|^{2} \nu(d z) d s \\
& \leqslant \varepsilon L \int_{|z| \leqslant 1}|z|^{2} \nu(d z) \int_{0}^{t}\left(1+\overline{\mathbb{E}}\left[\left|\mathcal{X}_{s-}^{\varepsilon, x}\right|^{2}\right]\right) d s \\
& \leqslant \varepsilon L \int_{|z| \leqslant 1}|z|^{2} \nu(d z) \int_{0}^{t}\left(1+2|x|^{2}+2 \overline{\mathbb{E}}\left[\left|\mathcal{X}_{s-}^{\varepsilon, x}-x\right|^{2}\right]\right) d s \\
& \leqslant \varepsilon L \int_{|z| \leqslant 1}|z|^{2} \nu(d z)\left(t\left(1+2|x|^{2}\right)+2 \int_{0}^{t} \overline{\mathbb{E}}\left[\left|\mathcal{X}_{s-}^{\varepsilon, x}-x\right|^{2}\right] d s\right) .
\end{aligned}
$$

Hence Gronwall's lemma yields for $t \in[0, \varepsilon], \varepsilon \in(0,1]$ and $C=L \int_{|z| \leqslant 1}|z|^{2} \nu(d z)$ a positive constant $C_{x}$ such that

$$
\overline{\mathbb{E}}\left[\left|\mathcal{X}_{t}^{\varepsilon, x}-x\right|^{2}\right] \leqslant \varepsilon C\left(1+2|x|^{2}\right) \int_{0}^{t} s \exp (\varepsilon 2 C(t-s)) d s \leqslant \varepsilon^{3} C_{x} .
$$

and hence for all positive $\varepsilon$ such that $\varepsilon^{3} C_{x}<\left(\frac{\rho}{2}\right)^{2}$ we have

$$
\sup _{t \in[0, \varepsilon]}\left|\overline{\mathbb{E}}\left[\mathcal{X}_{t}^{\varepsilon, x}\right]-x\right| \leqslant \frac{\rho}{2} \text {. }
$$

Therefore it follows for any $\lambda>0$

$$
\begin{aligned}
\overline{\mathbb{P}}\left(\sup _{t \in\left[0, \varepsilon \wedge \sigma^{\varepsilon}(x)\right]}\left|\mathcal{X}_{t}^{\varepsilon, x}-x\right| \geqslant \rho\right) \leqslant & \overline{\mathbb{P}}\left(\sup _{t \in\left[0, \varepsilon \wedge \sigma^{\varepsilon}(x)\right]}\left|\mathcal{X}_{t}^{\varepsilon, x}-\overline{\mathbb{E}}\left[\mathcal{X}_{t}^{\varepsilon, x}\right]\right| \geqslant \rho\right) \\
\leqslant & \overline{\mathbb{P}}\left(\sup _{t \in\left[0, \varepsilon \wedge \sigma^{\varepsilon}(x)\right]} \mid \mathcal{X}_{t}^{\varepsilon, x}-\overline{\mathbb{E}}\left[\mathcal{X}_{t}^{\varepsilon, x}\left|\geqslant \frac{\rho}{2}\right|\left[\mathcal{X}^{\varepsilon, x}-\overline{\mathbb{E}}\left[\mathcal{X}^{\varepsilon, x}\right]\right]_{\varepsilon \wedge \sigma^{\varepsilon}(x)} \leqslant \lambda\right)\right. \\
& +\overline{\mathbb{P}}\left(\left[\mathcal{X}^{\varepsilon, x}-\overline{\mathbb{E}}\left[\mathcal{X}^{\varepsilon, x}\right]\right]_{\varepsilon \wedge \sigma^{\varepsilon}(x)}>\lambda\right) .
\end{aligned}
$$

Theorem 3.3 of $\left[25\right.$ yields the following for the local martingale $\mathcal{X}_{t}^{\varepsilon, x}-\overline{\mathbb{E}}\left[\mathcal{X}_{t}^{\varepsilon, x}\right]$

$$
\overline{\mathbb{P}}\left(\sup _{t \in\left[0, \varepsilon \wedge \sigma^{\varepsilon}(x)\right]}\left|\mathcal{X}_{t}^{\varepsilon, x}-\overline{\mathbb{E}}\left[\mathcal{X}_{t}^{\varepsilon, x}\right]\right|>\frac{\rho}{2} \mid\left[\mathcal{X}^{\varepsilon, x}-\overline{\mathbb{E}}\left[\mathcal{X}^{\varepsilon, x}\right]\right]_{\varepsilon \wedge \sigma^{\varepsilon}(x)} \leqslant \lambda\right) \leqslant 2 \exp \left(-\frac{1}{2} \frac{\rho^{2}}{4 \lambda}\right) .
$$

For $\lambda=\lambda_{\varepsilon}:=\frac{\varepsilon}{\sqrt{|\ln (\varepsilon)|^{p}}}$ the latter estimate is asymptotically exponentially neglibile as $\varepsilon \rightarrow 0$. Hence it remains to prove that

$$
\overline{\mathbb{P}}\left(\left[\mathcal{X}^{\varepsilon, x}\right]_{\varepsilon \wedge \sigma^{\varepsilon}(x)}>\lambda_{\varepsilon}\right)
$$

is asymptotically exponentially negligible as $\varepsilon \rightarrow 0$.

1. Additive case: We start with the additive case $G(x, z)=z$ such that

$$
\left[\mathcal{X}^{\varepsilon, x}\right]_{t}=\varepsilon^{2} \int_{0}^{t} \int_{\sqrt{\varepsilon}|\ln (\varepsilon)|^{q}}|z|^{2} N^{\frac{1}{\varepsilon}}(d s, d z) .
$$

First we observe that due to Hypothesis E.1 $\iota(\varepsilon):=\int_{|z| \leqslant \sqrt{\varepsilon}|\ln \varepsilon|^{q}}|z|^{2} \nu(d z) \rightarrow 0$ as $\varepsilon \rightarrow 0$. Campbell's formula yields

$$
\left.\overline{\mathbb{E}}\left[\exp \left(\kappa\left[\mathcal{X}^{\varepsilon, x}\right]_{\varepsilon}\right)\right]=\exp \left(\varepsilon \int_{|z| \leqslant \sqrt{\varepsilon}|\ln \varepsilon|^{q}}\left(\exp \left(\kappa \varepsilon^{2}|z|^{2}\right)-1\right)\right) \frac{1}{\varepsilon} \nu(d z)\right) .
$$

If we choose $\kappa=\kappa_{\varepsilon}$ of order

$$
\kappa_{\varepsilon}=\frac{|\ln (\varepsilon)|^{p}}{\varepsilon^{2}}
$$

we obtain that as upper bound for the exponent

$$
\lim _{\varepsilon \rightarrow 0} \sup _{|z| \leqslant \sqrt{\varepsilon}|\ln \varepsilon|^{q}} \kappa_{\varepsilon} \varepsilon^{2}|z|^{2}=\frac{\varepsilon^{2}|\ln (\varepsilon)|^{p}\left(\sqrt{\varepsilon}|\ln \varepsilon|^{q}\right)^{2}}{\varepsilon^{2}}=\lim _{\varepsilon \rightarrow 0} \varepsilon|\ln \varepsilon|^{2 q+p}=0
$$


and for all $r \in(0,1)$ we have by Taylor's theorem $\exp (r)-1 \leqslant 2 r$. Therefore

$$
\begin{aligned}
\int_{|z| \leqslant \sqrt{\varepsilon}|\ln \varepsilon|^{q}}\left(\exp \left(\kappa_{\varepsilon} \varepsilon^{2}|z|^{2}\right)-1\right) \nu(d z) & \leqslant 2 \kappa_{\varepsilon} \varepsilon^{2} \int_{|z| \leqslant \varepsilon^{1 / 2}|\ln \varepsilon|^{q}}|z|^{2} \nu(d z) \\
& =2|\ln (\varepsilon)|^{p} \int_{|z| \leqslant \varepsilon^{1 / 2}|\ln \varepsilon|^{q}}|z|^{2} \nu(d z)
\end{aligned}
$$

and Hypothesis E.3 implies

$$
\limsup _{\varepsilon \rightarrow 0} \int_{|z| \leqslant \sqrt{\varepsilon}|\ln \varepsilon|^{q}}\left(\exp \left(\kappa_{\varepsilon} \varepsilon^{2}|z|^{2}\right)-1\right) \nu(d z) \leqslant \limsup _{\varepsilon \rightarrow 0} 2|\ln (\varepsilon)|^{p} \int_{|z| \leqslant \varepsilon^{1 / 2}|\ln \varepsilon|^{q}}|z|^{2} \nu(d z)<\infty .
$$

This implies for $\iota(\varepsilon)=\int_{|z| \leqslant \varepsilon^{1 / 2}|\ln \varepsilon|^{q}}|z|^{2} \nu(d z)$ the upper bound

$$
\limsup _{\varepsilon \rightarrow 0} \overline{\mathbb{E}}\left[\exp \left(\kappa_{\varepsilon}\left[\mathcal{X}^{\varepsilon, x}\right]_{\varepsilon}\right)\right] \leqslant \limsup _{\varepsilon \rightarrow 0} \exp \left(2|\ln (\varepsilon)|^{q} \iota(\varepsilon)\right) \leqslant \limsup _{\varepsilon \rightarrow 0} \exp \left(2|\ln (\varepsilon)|^{q} \iota(\varepsilon)\right)=E_{\infty}<\infty .
$$

Hence

$$
\begin{aligned}
\overline{\mathbb{P}}\left(\left[\mathcal{X}^{\varepsilon, x}\right]_{\varepsilon}>\lambda_{\varepsilon}\right) & \leqslant \exp \left(-\kappa_{\varepsilon} \frac{\varepsilon}{\sqrt{|\ln (\varepsilon)|^{p}}}\right) \exp \left(2|\ln (\varepsilon)|^{q} \iota(\varepsilon)\right) \\
& =\exp \left(-\frac{|\ln (\varepsilon)|^{p}}{\varepsilon} \frac{\varepsilon}{\sqrt{|\ln (\varepsilon)|}^{p}}\right) \exp \left(2|\ln (\varepsilon)|^{q} \iota(\varepsilon)\right) \leqslant \exp \left(-\frac{\sqrt{|\ln (\varepsilon)|^{p}}}{\varepsilon}\right) E_{\infty},
\end{aligned}
$$

which is asymptotically exponentially negligible as $\varepsilon \rightarrow 0$. Summing up we have obtained

$$
\begin{aligned}
& \varepsilon \ln \sup _{x \in D} \overline{\mathbb{P}}\left(\sup _{t \in[0, \varepsilon]}\left|X_{t}^{\varepsilon, x}-x\right| \geqslant \rho\right) \\
& \leqslant \varepsilon \ln \sup _{x \in D}\left(\overline{\mathbb{P}}\left(\sup _{t \in[0, \varepsilon]}\left|\mathcal{X}_{t}^{\varepsilon, x}-\mathbb{E}\left[\mathcal{X}_{t}^{\varepsilon, x}\right]\right| \geqslant \rho\right)+2 C e^{-\frac{|\ln (\varepsilon)|}{\varepsilon}}\right) \\
& \leqslant \varepsilon \ln \sup _{x \in D}\left(\overline{\mathbb{P}}\left(\sup _{t \in[0, \varepsilon]}\left|\mathcal{X}_{t}^{\varepsilon, x}-\mathbb{E}\left[\mathcal{X}_{t}^{\varepsilon, x}\right]\right| \geqslant \rho \mid\left[\mathcal{X}^{\varepsilon, x}\right]_{\varepsilon} \leqslant \frac{\varepsilon}{\sqrt{|\ln (\varepsilon)|^{p}}}\right)+\mathbb{P}\left(\left[\mathcal{X}^{\varepsilon, x}\right]_{\varepsilon}>\frac{\varepsilon}{\sqrt{|\ln (\varepsilon)|^{p}}}\right)\right. \\
& \left.\quad+2 C \exp \left(-\frac{|\ln (\varepsilon)|^{q}}{\varepsilon}\right)\right) \\
& \quad \\
& \leqslant \varepsilon \ln \sup _{x \in D}\left(2 \exp \left(-\frac{1}{8} \frac{\rho^{2} \sqrt{|\ln (\varepsilon)|^{q}}}{\varepsilon}\right)+\exp \left(-\frac{\sqrt{|\ln (\varepsilon)|^{p}}}{\varepsilon}\right) E_{\infty}+2 C \exp \left(-\Gamma \frac{|\ln (\varepsilon)|^{q}}{\varepsilon}\right)\right) \longrightarrow-\infty
\end{aligned}
$$

as $\varepsilon \rightarrow 0$.

2. Multiplicative case: For any $\varepsilon>0$ it follows

$$
\left[\mathcal{X}^{\varepsilon, x}-\overline{\mathbb{E}}\left[\mathcal{X}^{\varepsilon, x}\right]\right]_{\varepsilon}=\left[\mathcal{X}^{\varepsilon, x}\right]_{\varepsilon} \leqslant \varepsilon^{2} \int_{0}^{\varepsilon} \int_{0<|z| \leqslant \sqrt{\varepsilon}|\ln (\varepsilon)|^{q}}\left|G\left(\mathcal{X}_{s-}^{\varepsilon, x}, z\right)\right|^{2} N^{\frac{1}{\varepsilon}}(d s, d z) .
$$

By Hypothesis $[\mathrm{C}$ and due to the nonnegativity of the increments in the preceding quadratic variation we have

$$
\left[\mathcal{X}^{\varepsilon, x}\right]_{t} \leqslant \varepsilon^{2} 2 L^{2} \int_{0}^{t} \int_{0<|z| \leqslant \sqrt{\varepsilon}|\ln (\varepsilon)|^{q}}\left(1+\left|\mathcal{X}_{s-}^{\varepsilon, x}\right|^{2}\right)|z|^{2} N^{\frac{1}{\varepsilon}}(d s, d z)
$$

and

$$
\begin{aligned}
{\left[\mathcal{X}^{\varepsilon, x}\right]_{t \wedge \sigma^{\varepsilon}(x)} } & \leqslant \varepsilon^{2} 2 L^{2} \int_{0}^{t \wedge \sigma^{\varepsilon}(x)} \int_{0<|z| \leqslant \sqrt{\varepsilon}|\ln (\varepsilon)|^{q}} L\left(1+\left|\mathcal{X}_{s-}^{\varepsilon, x}\right|^{2}\right)|z|^{2} N^{\frac{1}{\varepsilon}}(d s, d z) \\
& \left.\leqslant 2 L^{2}\left(1+\operatorname{diam}(D)^{2}\right)\right) \varepsilon^{2} \int_{0}^{t} \int_{0<|z| \leqslant \sqrt{\varepsilon}|\ln (\varepsilon)|^{q}}|z|^{2} N^{\frac{1}{\varepsilon}}(d s, d z)
\end{aligned}
$$




$$
\left.\leqslant 2 L^{2}\left(1+\operatorname{diam}(D)^{2}\right)\right) \varepsilon^{2} \int_{0}^{t} \int_{0<|z| \leqslant \sqrt{\varepsilon}|\ln (\varepsilon)|^{q}}|z|^{2} N^{\frac{1}{\varepsilon}}(d s, d z) .
$$

As a consequence we may repeat the same reasoning of the additive case for $\left[\mathcal{X}^{\varepsilon, x}\right]_{\varepsilon \wedge \sigma^{\varepsilon}(x)}$ instead of $\left[\mathcal{X}^{\varepsilon, x}\right]_{\varepsilon}$, that is, for sufficiently small $\varepsilon$ we have the asymptotically exponentially negligible estimate

$$
\begin{aligned}
\overline{\mathbb{P}}\left(\left[\mathcal{X}^{\varepsilon, x}\right]_{\varepsilon \wedge \sigma^{\varepsilon}(x)}>\frac{\varepsilon}{\sqrt{|\ln (\varepsilon)|^{p}}}\right) & \leqslant e^{-\kappa_{\varepsilon} \frac{\varepsilon}{\sqrt{\left.\ln (\varepsilon)\right|^{p}}}} \exp \left(4 L^{2}\left(1+\operatorname{diam}(D)^{2}\right)|\ln (\varepsilon)|^{p} \iota(\varepsilon)\right) \\
& =e^{-\frac{\sqrt{|\ln (\varepsilon)|^{p}}}{\varepsilon}}\left(E_{\infty}\right)^{2 L^{2}\left(1+\operatorname{diam}(D)^{2}\right)} .
\end{aligned}
$$

Hence the virtually identical reasoning as in the additive case yields the desired result.

Lemma 23. Let $F \subset D^{c}$ closed. Then

$$
\lim _{\rho \rightarrow 0} \limsup _{\varepsilon \rightarrow 0} \varepsilon \ln \sup _{x \in B_{\rho}(0)} \overline{\mathbb{P}}\left(X_{\vartheta_{\rho}^{x}}^{\varepsilon, x} \in F\right) \leqslant-\inf _{z \in F} V(0, z) .
$$

Proof. Fix $\delta>0$ and $V_{F}(\delta):=\left(\inf _{z \in F} V(0, z)-\delta\right) \wedge \frac{1}{\delta}$. By definition of $V$ we have

$$
V(x, z) \leqslant V(x, y)+V(y, z) \text { for all } x, y, z \in \mathbb{R}^{d} .
$$

By Lemma 17 there is $\rho_{0}>0$ such that for $\rho \in\left(0, \rho_{0}\right]$ we have

$$
\inf _{z \in F, y \in \bar{B}_{\rho}(0)} V(y, z) \geqslant \inf _{z \in F} V(0, z)-\sup _{y \in \bar{B}_{\rho}(0)} V(0, y) \geqslant V_{F}(\delta) .
$$

Lemma 20 provides a constant $T>0$ such that for any $\rho \in\left(0, \rho_{0}\right]$

$$
\limsup _{\varepsilon \rightarrow 0} \varepsilon \ln \sup _{y \in \bar{B}_{\rho}(0)} \overline{\mathbb{P}}\left(\vartheta_{\rho}^{\varepsilon}(y)>T\right)<-V_{F}(\delta) .
$$

We consider the following subset of $\mathbb{D}\left([0, T], \mathbb{R}^{d}\right)$

$$
\mathcal{A}:=\left\{\varphi \in \mathbb{D}\left([0, T], \mathbb{R}^{d}\right) \mid \varphi(s) \in F \quad \text { for some } s \in[0, T]\right\} .
$$

We have that $\mathcal{A}$ is a closed set of $\mathbb{D}\left([0, T], \mathbb{R}^{d}\right)$ for the Skorokhod topology. For a proof we refer to Lemma 26 in the Appendix. Corollary 15 implies that there exists $\rho_{0}>0$ such that for $0<\rho<\rho_{0}$,

$$
\limsup _{\varepsilon \rightarrow 0} \varepsilon \ln \sup _{y \in \bar{B}_{\rho}(0)} \overline{\mathbb{P}}\left(X^{\varepsilon, y} \in \mathcal{A}\right) \leqslant-\inf _{y \in \bar{B}_{\rho}(0)} \inf _{\varphi \in \mathcal{A}} \mathbb{J}_{y, T}(\varphi) \leqslant-\inf _{y \in \bar{B}_{\rho}(0), z \in F} V(y, z) \leqslant-V_{F}(\delta) .
$$

Finally we have

$$
\begin{aligned}
& \limsup _{\varepsilon \rightarrow 0+} \varepsilon \ln \sup _{x \in \bar{B}_{\rho}(0)} \overline{\mathbb{P}}\left(X_{\vartheta_{\rho}}^{\varepsilon, x} \in F\right) \\
& \leqslant \limsup _{\varepsilon \rightarrow 0+} \varepsilon \ln \sup _{x \in \bar{B}_{\rho}(0)} \overline{\mathbb{P}}\left(\vartheta_{\rho}^{\varepsilon}(x)<\infty\right) \\
& \leqslant \limsup _{\varepsilon \rightarrow 0+} \varepsilon \ln \sup _{x \in \bar{B}_{\rho}(0)} \overline{\mathbb{P}}\left(\left\{\vartheta_{\rho}^{\varepsilon}(x)>T\right\} \cup\left\{\vartheta_{\rho}^{\varepsilon}(x) \leqslant T\right\}\right) \\
& \leqslant \limsup _{\varepsilon \rightarrow 0+} \ln \left(\sup _{y \in \bar{B}_{\rho}(0)} \overline{\mathbb{P}}\left(\vartheta_{\rho}^{\varepsilon}(y)>T\right)+\sup _{y \in \bar{B}_{\rho}(0)} \overline{\mathbb{P}}\left(X^{\varepsilon, y} \in \mathcal{A}\right)\right) \leqslant-V_{F}(\delta) .
\end{aligned}
$$

Sending $\delta \rightarrow 0$ finishes the proof.

Theorem 6. Let $\delta>0, x \in D$. Then we have

$$
\begin{aligned}
& \lim _{\varepsilon \rightarrow 0} \overline{\mathbb{P}}\left(\sigma^{\varepsilon}(x) \leqslant e^{\frac{\bar{V}-\delta}{\varepsilon}}\right)=0 \text { and } \\
& \liminf _{\varepsilon \rightarrow 0} \varepsilon \ln \overline{\mathbb{E}}\left[\sigma^{\varepsilon}(x)\right] \geqslant \bar{V}-\delta .
\end{aligned}
$$

Proof. The proof is organized in three consecutive steps. We start with the case $\bar{V}>0$. 
Step 1. Due to Hypothesis D there is $\rho^{\prime}>0$ such that $\bar{B}_{\rho^{\prime}}(0) \subset D$ and $\sup _{x \in B_{\rho^{\prime}}(0)}\langle b(x), n(x)\rangle<0$ and let $\rho>0$ such that $B_{\rho}(0) \subset B_{\rho^{\prime}}(0)$. We define recursively for any $x \in D$

$$
\begin{aligned}
\zeta_{0}^{x} & :=0 \quad \text { and for any } k \in \mathbb{N} \\
\vartheta_{k, \rho}^{x} & :=\inf \left\{t \geqslant \zeta_{k}^{x} \mid X_{t}^{\varepsilon, x} \in \bar{B}_{\rho}(0) \cup D^{c}\right\}, \\
\zeta_{k+1}^{x} & := \begin{cases}\infty, & \text { if } X_{\vartheta_{k, \rho}^{x}}^{\varepsilon, x} \in D^{c}, \\
\inf \left\{t \geqslant \vartheta_{k, \rho}^{x} \mid X_{t}^{\varepsilon, x} \in \bar{B}_{\rho^{\prime}}^{c}(0)\right\}, & \text { if } X_{\vartheta_{k, \rho}^{x}, x}^{\varepsilon,} \in \bar{B}_{\rho}(0) .\end{cases}
\end{aligned}
$$

By construction $\left(\zeta_{k}^{x}\right)_{k \in \mathbb{N}}$ and $\left(\vartheta_{k, \rho}^{x}\right)_{k \in \mathbb{N}}$ we have $\overline{\mathbb{P}}$-a.s. for all $k \in \mathbb{N}$

$$
\zeta_{k}^{x} \leqslant \vartheta_{k, \rho}^{x} \leqslant \zeta_{k+1}^{x} \leqslant \vartheta_{k+1, \rho}^{x} .
$$

Since $\rho^{\prime}>\rho$ we have that $\zeta_{k+1}^{x}>\vartheta_{k}^{x}$ if $X_{\vartheta_{k}^{x}}^{\varepsilon, x} \in \bar{B}_{\rho}(0)$. Hence $\left(\vartheta_{k, \rho}^{x}\right)_{k \in \mathbb{N}}$ is an increasing sequence of $\left(\mathcal{F}_{t}\right)$-stopping times. Since the process $\left(X_{t}^{\varepsilon, x}\right)_{t \geqslant 0}$ has the strong Markov property with respect to $\left(\mathcal{F}_{t}\right)_{t \geqslant 0}$ it follows that $\left(X_{\vartheta_{k, \rho}^{x}}^{\varepsilon, x}\right)_{k \in \mathbb{N}}$ is a Markov chain and $\sigma^{\varepsilon}(x)=\vartheta_{\ell, \rho}^{x}$ for some (random) $\ell \in \mathbb{N}$ with the convention $X_{\vartheta_{\ell, \rho}^{x}}^{\varepsilon, x}:=X_{\sigma^{\varepsilon}(x)}^{\varepsilon, \rho}$ if $\vartheta_{\ell, \rho}^{x}=\infty$.

Claim 2. For any $x \in D, \varepsilon>0, T>0$ and $k \in \mathbb{N}$ arbitrary it follows

$$
\left\{\sigma^{\varepsilon}(x) \leqslant T\right\} \subset\left\{\sigma^{\varepsilon}(x)=\vartheta_{0}^{x, \rho}\right\} \cup \bigcup_{m=1}^{k}\left\{\sigma^{\varepsilon}(x)=\vartheta_{m, \rho}^{x}\right\} \cup\left\{\zeta_{m}^{x}-\vartheta_{m-1, \rho}^{x} \leqslant T \wedge \sigma^{\varepsilon}(x)\right\} .
$$

Proof. Fix $k \in \mathbb{N}$ such that $\sigma^{\varepsilon}(x)>\vartheta_{k, \rho}^{x}$ and $\zeta_{m}^{x}-\vartheta_{m-1, \rho}^{x}>T \wedge \sigma^{\varepsilon}(x)$ for every $m \in\{1, \ldots, k\}$. It follows

$$
\sigma^{\varepsilon}(x)>\vartheta_{k, \rho}^{x}=\sum_{m=1}^{k}\left(\vartheta_{m, \rho}^{x}-\vartheta_{m-1, \rho}^{x}\right)+\vartheta_{0, \rho}^{x} \geqslant \sum_{m=1}^{k}\left(\zeta_{m}^{x}-\vartheta_{m-1, \rho}^{x}\right)>k\left(T \wedge \sigma^{\varepsilon}(x)\right) .
$$

Contraposition of the preceding inclusion of events and eliminating redundancies yield

$$
\begin{aligned}
\left\{\sigma^{\varepsilon}(x) \leqslant k T\right\} & \subset\left\{\sigma^{\varepsilon}(x) \leqslant \vartheta_{0}^{x, \rho}\right\} \cup \bigcup_{m=1}^{k}\left\{\sigma^{\varepsilon}(x) \leqslant \vartheta_{m, \rho}^{x}\right\} \cup\left\{\zeta_{m}^{x}-\vartheta_{m-1, \rho}^{x} \leqslant T \wedge \sigma^{\varepsilon}(x)\right\} \\
& =\left\{\sigma^{\varepsilon}(x)=\vartheta_{0}^{x, \rho}\right\} \cup \bigcup_{m=1}^{k}\left\{\sigma^{\varepsilon}(x)=\vartheta_{m, \rho}^{x}\right\} \cup\left\{\zeta_{m}^{x}-\vartheta_{m-1, \rho}^{x} \leqslant T \wedge \sigma^{\varepsilon}(x)\right\} .
\end{aligned}
$$

Fix $\delta>0$. We set $k(\varepsilon):=\left\lfloor\frac{1}{T(\varepsilon)} e^{\frac{\bar{v}-\delta}{\varepsilon}}\right\rfloor+1$ for $T(\varepsilon)=\varepsilon$. For any $x \in D$ this yields

$$
\overline{\mathbb{P}}\left(\sigma^{\varepsilon}(x) \leqslant e^{\frac{\bar{v}-\delta}{\varepsilon}}\right) \leqslant \overline{\mathbb{P}}\left(\sigma^{\varepsilon}(x) \leqslant k(\varepsilon)\left(T(\varepsilon) \wedge \sigma^{\varepsilon}(x)\right)\right) .
$$

The inclusion of events (58) implies that

$$
\left.\overline{\mathbb{P}}\left(\sigma^{\varepsilon}(x) \leqslant k(\varepsilon) T\right) \leqslant \overline{\mathbb{P}}\left(\sigma^{\varepsilon}(x)=\vartheta_{0}^{x, \rho}\right)+\sum_{m=1}^{k(\varepsilon)}\left(\overline{\mathbb{P}}\left(\sigma^{\varepsilon}(x)=\vartheta_{m, \rho}^{x}\right)+\overline{\mathbb{P}}\left(\zeta_{m}^{x}-\vartheta_{m-1, \rho}^{x} \leqslant T(\varepsilon) \wedge \sigma^{\varepsilon}(x)\right)\right)\right) .
$$

Step 2. Using Lemma 23 there exists $\varepsilon_{0}>0$ and $\rho>0$ such that for $\varepsilon \in\left(0, \varepsilon_{0}\right]$ we have

$$
\limsup _{\varepsilon \rightarrow 0} \varepsilon \ln \sup _{x \in \bar{B}_{\rho}(0)} \overline{\mathbb{P}}\left(X_{\vartheta_{\rho}^{x}}^{\varepsilon, x} \in D^{c}\right) \leqslant-\bar{V}+\frac{\delta}{2} .
$$


Let $0<\rho<\rho_{0}$ satisfy Step 1 such that $0<\rho^{\prime}-\rho<\rho_{0}$ for some $\rho_{0}>0$ fixed below, $x \in D$ and $m \geqslant 1$. Since $\sigma^{\varepsilon}(x)<\infty \overline{\mathbb{P}}$-a.s. and due to the strong Markov property we have

$$
\begin{aligned}
\sup _{x \in D} \overline{\mathbb{P}}\left(\sigma^{\varepsilon}(x)=\vartheta_{m, \rho}^{x}\right) & \leqslant \sup _{x \in D} \overline{\mathbb{P}}\left(X_{\vartheta_{m, \rho}^{x}}^{\varepsilon, x} \in D^{c} ; \vartheta_{m, \rho}^{x}<\infty\right) \\
& \leqslant \sup _{x \in D} \overline{\mathbb{P}}\left(X_{\vartheta_{m-1, \rho}^{x}, x}^{\varepsilon,} \in \bar{B}_{\rho}(0) ; X_{\vartheta_{m, \rho}^{x}}^{\varepsilon, x} \in D^{c}\right) \leqslant \sup _{y \in \bar{B}_{\rho}(0)} \overline{\mathbb{P}}\left(X_{\vartheta_{\rho}^{y}}^{\varepsilon} \in D^{c}\right) .
\end{aligned}
$$

Lemma 23 yields for any $m \geqslant 1$ and $\delta>0$ that there exists $\rho_{0}>0$ such that for any $\rho<\rho_{0}$ we have

$$
\limsup _{\varepsilon \rightarrow 0} \varepsilon \ln \overline{\mathbb{P}}\left(\sigma^{\varepsilon}(x)=\vartheta_{m, \rho}^{x}\right) \leqslant-\bar{V}+\frac{\delta}{2} .
$$

For the constant $c=\bar{V}-\frac{\delta}{2}>0$ and $\rho_{0}>0$ such that for $\rho^{\prime}-\rho \leqslant \rho_{0}$ Lemma 22 implies

$$
\begin{aligned}
\sup _{y \in D} \overline{\mathbb{P}}\left(\zeta_{k}^{y}-\vartheta_{k-1, \rho}^{y} \leqslant T(\varepsilon) \wedge \sigma^{\varepsilon}(x)\right) & \leqslant \sup _{y \in B_{\rho}(0)} \overline{\mathbb{P}}\left(\sup _{t \in\left[0, T(\varepsilon) \wedge \sigma^{\varepsilon}(x)\right]}\left|X_{t}^{\varepsilon, y}-y\right| \geqslant \rho^{\prime}-\rho\right) \\
& \leqslant \sup _{y \in D} \overline{\mathbb{P}}\left(\sup _{t \in\left[0, T(\varepsilon) \wedge \sigma^{\varepsilon}(x)\right]}\left|X_{t}^{\varepsilon, y}-y\right| \geqslant \rho^{\prime}-\rho\right) .
\end{aligned}
$$

Therefore, for the constant $c=\bar{V}-\frac{\delta}{2}>0$ fixed above we obtain

$$
\limsup _{\varepsilon \rightarrow 0} \varepsilon \ln \sup _{y \in D} \overline{\mathbb{P}}\left(\sup _{t \in\left[0, T(\varepsilon) \wedge \sigma^{\varepsilon}(x)\right]}\left|X^{\varepsilon, y}-y\right| \geqslant \rho^{\prime}-\rho\right)<-\left(\bar{V}-\frac{\delta}{2}\right) .
$$

Hence, combining (59)- (64) yields for any $k \in \mathbb{N}$ and $x \in D$ that

$$
\begin{aligned}
& \overline{\mathbb{P}}\left(\sigma^{\varepsilon}(x) \leqslant k(\varepsilon)\left(T(\varepsilon) \wedge \sigma^{\varepsilon}(x)\right)\right) \\
& \leqslant \overline{\mathbb{P}}\left(\sigma^{\varepsilon}(x)=\vartheta_{0, \rho}^{x}\right)+\sum_{m=1}^{k(\varepsilon)}\left(\overline{\mathbb{P}}\left(\sigma^{\varepsilon}(x)=\vartheta_{m, \rho}^{x}\right)+\overline{\mathbb{P}}\left(\zeta_{m}^{x}-\vartheta_{m-1, \rho}^{x} \leqslant T(\varepsilon) \wedge \sigma^{\varepsilon}(x)\right)\right) \\
& \leqslant \overline{\mathbb{P}}\left(\sigma^{\varepsilon}(x)=\vartheta_{0, \rho}^{x}\right)+2 k(\varepsilon) e^{-\frac{\bar{V}-\frac{\delta}{2}}{\varepsilon}} .
\end{aligned}
$$

Due to Lemma 21 we have for all $x \in D$ the desired result

$$
\overline{\mathbb{P}}\left(\sigma^{\varepsilon}(x) \leqslant e^{\frac{\bar{V}-\delta}{\varepsilon}}\right) \leqslant \overline{\mathbb{P}}\left(\sigma^{\varepsilon}(x) \leqslant k(\varepsilon) T(\varepsilon)\right) \leqslant \overline{\mathbb{P}}\left(X_{\vartheta_{0, \rho}^{x}}^{\varepsilon, x} \notin \bar{B}_{\rho}(0)\right)+\frac{2}{T(\varepsilon)} e^{-\frac{\delta}{2 \varepsilon}} \rightarrow 0 \quad \text { as } \varepsilon \rightarrow 0 .
$$

Chebyshev's inequality implies for $\varepsilon>0$ sufficiently small that

$$
\overline{\mathbb{E}}\left[\sigma^{\varepsilon}(x)\right] \geqslant e^{\frac{\bar{v}-\delta}{\varepsilon}} \overline{\mathbb{P}}\left(\sigma^{\varepsilon}(x) \geqslant e^{\frac{\bar{V}-\delta}{\varepsilon}}\right) \geqslant \frac{1}{2} e^{\frac{\bar{V}-\delta}{\varepsilon}} .
$$

This finishes the proof for the case $\bar{V}>0$.

Step 3. We treat the case $\bar{V}=0$. Let $\delta>0$ and $x \in D$. Choose $\rho>0$ such that $\bar{B}_{\rho}(0) \subset D$. Assume $c>0$. By the strong Markov property we can choose $\varepsilon_{0} \in(0,1]$ such that for $\varepsilon \in\left(0, \varepsilon_{0}\right]$

$$
\overline{\mathbb{P}}\left(\sigma^{\varepsilon}(x)>e^{-\frac{\delta}{\varepsilon}}\right) \geqslant \overline{\mathbb{P}}\left(X_{\vartheta_{\rho}^{x}}^{\varepsilon, x} \in \bar{B}_{\rho}(0)\right) \inf _{y \in B_{\rho}(0)} \overline{\mathbb{P}}\left(\sup _{t \in\left[0, \varepsilon \wedge \sigma^{\varepsilon}(x)\right]}\left|X^{\varepsilon, y}-y\right| \leqslant \rho\right) .
$$

Lemma 21] and Lemma 22 imply that the right-hand side of (67) converges to 1 as $\varepsilon \rightarrow 0$. This finishes the proof. 


\section{The exit location in Theorem 3:}

Remark 24. The proof of the statement 2. of Theorem 3 goes along the same line of reasoning as in the Brownian case and is extensively documented in the literature in different settings. We refer the reader for example to Theorem 4.2.4 in 29 to a a more general setting for the deterministic dynamical system (2) with an additive Brownian perturbation and to Theorem 5.7.11 in 22 for a multiplicative Brownian perturbation of (2). Our result is obtained with analogous arguments used to prove the second statement of Theorem 2.4.6 in [55] (pp. 88-90). Therefore we omit the proof and refer the reader to [55].

\section{Appendix}

\subsection{Preliminaries for the proof of the LDP}

\subsubsection{Proof of Lemma 9}

Let $\nu \in \mathfrak{M}$ satisfy Hypothesis $\mathrm{E}$

Step 1. We start with the proof of (24). Let $g \in \mathfrak{S}^{M}$. We have the decomposition

$$
\int_{0}^{T} \int_{\mathbb{R}^{d} \backslash\{0\}}|z|^{2} g(s, z) \nu(d z) d s \leqslant \int_{0}^{T} \int_{0<|z| \leqslant 1}|z|^{2} g(s, z) \nu(d z) d s+\int_{0}^{T} \int_{|z|>1}|z|^{2} g(s, z) \nu(d z) d s .
$$

The first integral on the right hand side of (68) is estimated as follows. Young's inequality reads for any $a, b \geqslant 0$ that $a b \leqslant e^{a}+b \ln b-b$. This implies that

$$
\begin{aligned}
\int_{0}^{T} \int_{0<|z| \leqslant 1}|z|^{2} g(s, z) \nu(d z) d s & \leqslant \int_{0}^{T} \int_{0<|z| \leqslant 1}|z|^{2}(e+\ell(g(s, z))) \nu(d z) d s \\
& \leqslant e T c_{\nu}^{2}+\int_{0}^{T} \int_{0<|z| \leqslant 1}|z|^{2} \ell(g(s, z)) \nu(d z) d s \leqslant e T c_{\nu}^{2}+M<\infty
\end{aligned}
$$

since $\nu$ is a Lévy measure $\left(c_{\nu}^{2}:=\int_{0<|z| \leqslant 1}|z|^{2}<\infty\right)$. For the second integral in the right hand side of (68) we estimate

$$
\int_{0}^{T} \int_{|z| \geqslant 1}|z|^{2} g(s, z) \nu(d z) d s \leqslant T\left(\int_{|z| \geqslant 1} e^{\Gamma|z|^{2}} \nu(d z)\right)+\frac{1}{\Gamma} \int_{0}^{T} \int_{|z| \geqslant 1} \ell(g(s, z)) \nu(d z) d z<\infty
$$

since $\nu$ satisfies the integrability assumption (5) and $g \in \mathfrak{S}^{M}$. Combining (68), (69) and (70) yields (24).

Step 2. We fix $M>0, g \in \mathfrak{S}^{M}$ and $I \subset[0, T]$ a measurable set. Remark 3.3. in [14 states for any $\beta>0$ that

$$
|x-1| \leqslant c_{1}(\beta) \ell(x) \quad \text { if }|x-1| \geqslant \beta \text { and }|x-1|^{2} \leqslant c_{2}(\beta) \ell(x) \quad \text { if }|x-1| \leqslant \beta,
$$

for some $c_{1}(\beta), c_{2}(\beta)>0$ where $c_{1}(\beta) \rightarrow 0$ as $\beta \rightarrow 0$. Let $\beta>0$ and consider the measurable set

$$
E_{\beta}:=\left\{(s, z) \in I \times \mathbb{R}^{d} \backslash\{0\}|| g(s, z)-1 \mid \leqslant \beta\right\} .
$$

We apply the following version of Young's inequality: $a b \leqslant e^{\sigma a}+\frac{1}{\sigma} \ell(b)$ for any $a, b>0$ and $\sigma \geqslant 1$, and obtain for any $\beta>0$ and $\sigma \geqslant 1$

$$
\int_{I} \int_{\mathbb{R}^{d} \backslash\{0\}}|z \| g(s, z)-1| \nu(d z) d s \leqslant \int_{E_{\beta}}|z||g(s, z)-1| \nu(d z) d s+\int_{E_{\beta}^{c}}|z||g(s, z)-1| \nu(d z) d s
$$




$$
\begin{aligned}
\leqslant & \left(\int_{I} \int_{\mathbb{R}^{d} \backslash\{0\}}|z|^{2} \nu(d z) d s\right)^{\frac{1}{2}} \sqrt{c_{2}(\beta)} \sqrt{M}+\int_{E_{\beta}^{c} \cap\{|z|>1\}}|z|(g(s, z)-1) \nu(d z) d s \\
& +\int_{E_{\beta}^{c} \cap\{0<|z|<1\}}|z|(g(s, z)-1) \nu(d z) d s \\
\leqslant & \sqrt{|I| C_{1} c_{2}(\beta) M}+|I|\left(C_{2}+C_{3}\right)+\frac{M}{\sigma}+c_{1}(\beta) M .
\end{aligned}
$$

In the preceding estimate we used

$$
C_{1}:=\int_{\mathbb{R}^{d} \backslash\{0\}}|z|^{2} \nu(d z), \quad C_{2}:=\int_{|z|>1} e^{\sigma|z|} \nu(d z) \quad \text { and } C_{3}:=\int_{|z|>1}|z| \nu(d z),
$$

all of which are finite since $\nu$ is a Lévy measure on $\left(\mathbb{R}^{d} \backslash\{0\}, \mathcal{B}\left(\mathbb{R}^{d} \backslash\{0\}\right)\right)$ and satisfies (5). Choosing $I=[0, T]$ in (72) proves (25).

Step 3. In order to prove (26) let us fix $\delta^{\prime}>0$ arbitrary and $t, t^{\prime} \in[0, T]$ such that $\left|t-t^{\prime}\right|<\delta$ with $\delta>0$ fixed below. Let $\beta>0$ be sufficiently large such that $c_{1}(\beta) M<\frac{\delta^{\prime}}{4}$ and $\sigma>\frac{4 M}{\delta^{\prime}}$. Finally fix

$\delta>0$ such that $\delta<\frac{\delta^{\prime}}{4\left(C_{2}+C_{3}\right)} \wedge \frac{\left(\delta^{\prime}\right)^{2}}{16\left(C_{1} c_{2}(\beta) M\right)^{2}}$. For the choice of $\beta, \sigma \geqslant 1$ and $\delta>0$ as above, one has that (72) implies

$$
\int_{t}^{t^{\prime}} \int_{\mathbb{R}^{d} \backslash\{0\}}|z||g(s, z)-1| \nu(d z) d s<\delta^{\prime} .
$$

This finishes the proof of (26).

\subsubsection{Proof of Proposition 10}

For convenience of notation we drop the dependence of $\tilde{X}^{\varepsilon, x}$ on $x$. For every $\varepsilon>0$ let $\mathcal{R}(\varepsilon)>0$ such that $\mathcal{R}(\varepsilon) \rightarrow \infty$ and $\varepsilon \mathcal{R}^{2}(\varepsilon) \rightarrow 0$ as $\varepsilon \rightarrow 0$, for example $\mathcal{R}(\varepsilon):=|\ln \varepsilon|, \varepsilon>0$. By definition of $\tilde{\tau}_{\mathcal{R}(\varepsilon)}^{\varepsilon}$ in (28) it follows

$$
\overline{\mathbb{P}}\left(\sup _{t \in[0, T]}\left|\tilde{X}_{t}^{\varepsilon}\right|>\mathcal{R}(\varepsilon)\right) \leqslant \overline{\mathbb{P}}\left(\sup _{t \in\left[0, \tilde{\tau}_{\mathcal{R}(\varepsilon)}^{\varepsilon} \wedge T\right]}\left|\tilde{X}_{t}^{\varepsilon}\right|>\mathcal{R}(\varepsilon)\right) .
$$

We observe that for every $\varepsilon>0$ the process $\left(\tilde{X}_{t}^{\varepsilon}\right)_{t \in[0, T]}$ is a locally square integrable martingale. Therefore we use the Bernstein-type inequality given by Theorem 3.3 of [25] and infer for some parameter $\lambda=\lambda_{\varepsilon}>0$ that is fixed below

$$
\begin{aligned}
\overline{\mathbb{P}}\left(\sup _{t \in\left[0, \tilde{\tau}_{\mathcal{R}(\varepsilon)}^{\varepsilon} \wedge T\right]}\left|\tilde{X}_{t}^{\varepsilon}\right|>\mathcal{R}(\varepsilon)\right) & \leqslant \overline{\mathbb{P}}\left(\sup _{t \in\left[0, \tilde{\tau}_{\mathcal{R}(\varepsilon)}^{\varepsilon} \wedge T\right]}\left|\tilde{X}_{t}^{\varepsilon}\right|>\mathcal{R}(\varepsilon),\left[\tilde{X}^{\varepsilon}\right]_{\tilde{\tau}_{\mathcal{R}(\varepsilon)}^{\varepsilon} \wedge T} \leqslant \lambda\right)+\overline{\mathbb{P}}\left(\left[\tilde{X}^{\varepsilon}\right]_{\tilde{\tau}_{\mathcal{R}(\varepsilon)}^{\varepsilon} \wedge T}>\lambda\right) \\
& \leqslant 2 \exp \left(-\frac{1}{2} \frac{\mathcal{R}^{2}(\varepsilon)}{\lambda}\right)+\overline{\mathbb{P}}\left(\left[\tilde{X}^{\varepsilon}\right]_{\tilde{\tau}_{\mathcal{R}(\varepsilon)}^{\varepsilon}}>\lambda\right)
\end{aligned}
$$

For every $\varepsilon>0$ the quadratic variation of the process $\left(\tilde{X}^{\varepsilon}\right)_{t \in[0, T]}$ is given for every $t \in[0, T]$ by

$$
\left[\tilde{X}^{\varepsilon}\right]_{t}=\varepsilon^{2} \int_{0}^{t} \int_{\mathbb{R}^{d} \backslash\{0\}}\left|G\left(\tilde{X}_{s-}^{\varepsilon}\right)\right|^{2}|z|^{2} N^{\frac{\varphi_{\varepsilon}}{\varepsilon}}(d s, d z) .
$$

Due to Hypothesis $[$ and Chebyshev's inequality the second probability of the last term of (73) is estimated for $\varepsilon \in\left(0, \varepsilon_{0}\right]$ with $\varepsilon_{0}>0$ small enough as follows

$$
\overline{\mathbb{P}}\left(\left[\tilde{X}^{\varepsilon}\right]_{\tilde{\tau}_{\mathcal{R}(\varepsilon)}^{\varepsilon} \wedge T}>\lambda\right) \leqslant \overline{\mathbb{P}}\left(\varepsilon^{2} \int_{0}^{\tilde{\tau}_{\mathcal{R}(\varepsilon)}^{\varepsilon} \wedge T} \int_{\mathbb{R}^{d} \backslash\{0\}}\left|G\left(\tilde{X}_{s-}^{\varepsilon}, z\right)\right|^{2} N^{\frac{\varphi_{\varepsilon}}{\varepsilon}}(d s, d z)>\lambda\right)
$$




$$
\begin{aligned}
& \leqslant \overline{\mathbb{P}}\left(2 L^{2} \varepsilon^{2}\left(1+\mathcal{R}^{2}(\varepsilon)\right) \int_{0}^{T} \int_{\mathbb{R}^{d} \backslash\{0\}}|z|^{2} \varphi_{\varepsilon}(s, z) \nu(d z) d s>\lambda\right) \\
& \leqslant \frac{2 L^{2} \bar{C} \varepsilon\left(1+\mathcal{R}^{2}(\varepsilon)\right)}{\lambda}
\end{aligned}
$$

where $\bar{C}:=\sup _{g \in \mathfrak{S}^{M}} \int_{0}^{T} \int_{\mathbb{R}^{d} \backslash\{0\}}|z|^{2} g(s, z) \nu(d z) d s<\infty$ by (24) of Lemma 9. Set $\lambda=\lambda_{\varepsilon}=\mathcal{R}(\varepsilon), \varepsilon>0$. Combining (73) and (74) yields some $C>0$ and $\varepsilon_{0}>0$ such that $\varepsilon<\varepsilon_{0}$ implies

$$
\overline{\mathbb{P}}\left(\sup _{t \in[0, T]}\left|\tilde{X}_{s}^{\varepsilon}\right|>\mathcal{R}(\varepsilon)\right) \leqslant 2 e^{-\frac{1}{2} \mathcal{R}(\varepsilon)}+C \varepsilon \mathcal{R}(\varepsilon),
$$

which converges to 0 as $\varepsilon \rightarrow 0$.

\subsubsection{Proof of Proposition 11}

For every $\varepsilon>0$ let $\mathcal{R}(\varepsilon)>0$ fixed as in the statement of Proposition 10, i.e. such that $\mathcal{R}(\varepsilon) \rightarrow \infty$ and $\varepsilon \mathcal{R}^{2}(\varepsilon) \rightarrow 0$ as $\varepsilon \rightarrow 0$ and $\tilde{\tau}_{\mathcal{R}(\varepsilon)}^{\varepsilon}$ in (28). We drop the dependence on $x \in \mathbb{R}^{d}$ wherever possible without confusion. Ito's formula, Hypotheses $\mathrm{A}$, B and $\mathrm{C}$ and the inequality (24) imply on the event $\left\{T<\tilde{\tau}_{\mathcal{R}(\varepsilon)}^{\varepsilon}\right\}$ for any $t \in[0, T], \varepsilon<\varepsilon_{1}$ with $\varepsilon_{1}>0$ sufficiently small and $\overline{\mathbb{P}}$-a.s. that the following holds

$$
\begin{aligned}
\left|\tilde{X}_{t}^{\varepsilon}\right|^{2} \leqslant & |x|^{2}+2 \int_{0}^{t} \int_{\mathbb{R}^{d} \backslash\{0\}}\left\langle G\left(\tilde{X}_{s}^{\varepsilon}\right) z, \tilde{X}_{s}^{\varepsilon}\right\rangle\left(\varphi_{\varepsilon}(s, z)-1\right) \nu(d z) d s \\
& +M_{1}^{\varepsilon}(t)+M_{2}^{\varepsilon}(t)+\varepsilon \int_{0}^{t} \int_{\mathbb{R}^{d} \backslash\{0\}}\left|G\left(\tilde{X}_{s}^{\varepsilon}\right) z\right|^{2} \varphi^{\varepsilon}(s, z) \nu(d z) d s \\
\leqslant & |x|^{2}+2 L^{2} C_{0}+2 L^{2} \int_{0}^{t}\left|\tilde{X}_{s}^{\varepsilon}\right|^{2} \Theta^{\varepsilon}(s) d s+M_{1}^{\varepsilon}(t)+M_{2}^{\varepsilon}(t)+2 \varepsilon L^{2}\left(1+\mathcal{R}^{2}(\varepsilon)\right) C_{1} \\
\leqslant & 2|x|^{2}+2 L^{2} C_{0}+2 L^{2} \int_{0}^{t}\left|\tilde{X}_{s}^{\varepsilon}\right|^{2} \Theta^{\varepsilon}(s) d s+M_{1}^{\varepsilon}(t)+M_{2}^{\varepsilon}(t),
\end{aligned}
$$

where for any $t \in[0, T]$ and $\varepsilon>0$ we denote the processes

$$
\left\{\begin{array}{l}
M_{1}^{\varepsilon}(s):=\int_{0}^{t} \int_{\mathbb{R}^{d} \backslash\{0\}} \varepsilon^{2}\left|G\left(\tilde{X}_{s-}^{\varepsilon}\right) z\right|^{2} \tilde{N}^{\frac{1}{\varepsilon} \varphi^{\varepsilon}}(d s, d z) \\
M_{2}^{\varepsilon}(s):=\int_{0}^{t} \int_{\mathbb{R}^{d} \backslash\{0\}} 2 \varepsilon\left\langle G\left(\tilde{X}_{s-}^{\varepsilon}\right) z, \tilde{X}_{s-}^{\varepsilon}\right\rangle \tilde{N}^{\frac{1}{\varepsilon} \varphi^{\varepsilon}}(d s, d z)
\end{array}\right.
$$

and the constants

$$
\left\{\begin{array}{l}
C_{0}:=\sup _{g \in \mathfrak{S}^{M}} \int_{0}^{T} \int_{\mathbb{R}^{d} \backslash\{0\}}|z||g(s, z)-1| \nu(d z) d s<\infty, \quad \text { due to (25), } \\
C_{1}:=\sup _{g \in \mathfrak{S}^{M}} \int_{0}^{T} \int_{\mathbb{R}^{d} \backslash\{0\}}|z|^{2} g(s, z) \nu(d z) d s<\infty, \quad \text { due to (24). }
\end{array}\right.
$$

In addition, $\Theta^{\varepsilon}(s):=\int_{\mathbb{R}^{d} \backslash\{0\}}|z|\left|\varphi^{\varepsilon}(s, z)-1\right| \nu(d z) d s$ is such that $\int_{0}^{T} \Theta^{\varepsilon}(s, z) d s<\infty$ due to (25). Gronwall's lemma yields a constant $C_{2}>0$ such that for every $\varepsilon>0$ small enough, the event $\left\{T>\tilde{\tau}_{\mathcal{R}(\varepsilon)}^{\varepsilon}\right\}$ implies

$$
\sup _{t \in[0, T]}\left|\tilde{X}_{t}^{\varepsilon}\right|^{2} \leqslant C_{2} e^{2 L^{2} \int_{0}^{T} \Theta^{\varepsilon}(s) d s}\left(1+\sup _{t \in[0, T]}\left|M_{1}^{\varepsilon}(t)\right|+\sup _{t \in[0, T]}\left|M_{2}^{\varepsilon}(t)\right|\right) .
$$


The Burkholder-Davis-Gundy and the Jensen inequalities yield some $C_{3}>0$ such that

$$
\begin{aligned}
\overline{\mathbb{E}}\left[\sup _{t \in\left[0, \tilde{\tau}_{\mathcal{R}(\varepsilon)}^{\varepsilon}\right]}\left|M_{1}^{\varepsilon}(s)\right|\right] & \leqslant C_{3} \overline{\mathbb{E}}\left[\left(\int_{0}^{\tilde{\tau}_{\mathcal{R}(\varepsilon)}^{\varepsilon}} \int_{\mathbb{R}^{d} \backslash\{0\}} \varepsilon^{4}\left|G\left(\tilde{X}_{s-}^{\varepsilon}, z\right)\right|^{2} N^{\frac{1}{\varepsilon} \varphi^{\varepsilon}}(d s, d z)\right)^{\frac{1}{2}}\right] \\
& \leqslant C_{3} \varepsilon^{2} \mathcal{R}(\varepsilon) \sqrt{\overline{\mathbb{E}}\left[\int_{0}^{T} \int_{\mathbb{R}^{d} \backslash\{0\}}|z|^{2} N^{\frac{1}{\varepsilon}} \varphi^{\varepsilon}(d s, d z)\right]} \\
& \leqslant C_{3} \frac{\varepsilon^{2} \mathcal{R}^{2}(\varepsilon)}{\sqrt{\varepsilon}} \sqrt{C_{1}} \rightarrow 0 \quad \text { as } \varepsilon \rightarrow 0 .
\end{aligned}
$$

Analogously it is shown that

$$
\overline{\mathbb{E}}\left[\sup _{t \in\left[0, \tilde{\tau}_{\mathcal{R}(\varepsilon)}^{\varepsilon}\right]}\left|M_{2}^{\varepsilon}(t)\right|\right] \rightarrow 0, \quad \text { as } \varepsilon \rightarrow 0 .
$$

Hence from (76), (77) and (78) it follows for some $C_{4}>0$ and every $\varepsilon>0$ small enough that

$$
\overline{\mathbb{E}}\left[\sup _{t \in\left[0, \tilde{\tau}_{\mathcal{R}(\varepsilon)}^{\varepsilon}\right]}\left|\tilde{X}_{t}^{\varepsilon}\right|^{2}\right] \leqslant C_{4}\left(1+\overline{\mathbb{E}}\left[\sup _{t \in\left[0, \tilde{\tau}_{\mathcal{R}(\varepsilon)}^{\varepsilon}\right]}\left|M_{1}^{\varepsilon}(t)\right|\right]+\overline{\mathbb{E}}\left[\sup _{t \in\left[0, \tilde{\tau}_{\mathcal{R}(\varepsilon)}^{\varepsilon}\right]}\left|M_{2}^{\varepsilon}(t)\right|\right]\right)<\infty .
$$

This finishes the proof.

\subsection{Proof of Lemma 17}

The statements (41) and (42) are a consequence of the following fact. For any fixed $M>0$ and $g \in \mathfrak{S}^{M}$ Proposition [16 ensures for any $\rho>0$ some $\xi(\rho)>0$ such that $\xi(\rho) \rightarrow 0$ as $\rho \rightarrow 0$ and $\Phi \in C\left([0, \xi(\rho)], \mathbb{R}^{d}\right)$ solving (40). Since for the function $\ell(b)=b \ln b-b+1, b \geqslant 0$ we have

$$
\int_{0}^{T} \int_{\mathbb{R}^{d} \backslash\{0\}} \ell(g(s, z)) \nu(d z) d s \leqslant M,
$$

the monotone convergence theorem yields

$$
\lim _{\rho \rightarrow 0} \int_{0}^{\xi(\rho)} \int_{\mathbb{R}^{d} \backslash\{0\}} \ell(g(s, z)) \nu(d z) d s=\int_{0}^{T} \int_{\mathbb{R}^{d} \backslash\{0\}} \lim _{\rho \rightarrow 0} \mathbf{1}_{[0, \xi(\rho)]}(s) \ell(g(s, z)) \nu(d z) d s=0 .
$$

Hence for any $\delta>0$ there is $\rho_{0}>0$ small enough such that $\rho \in\left(0, \rho_{0}\right]$ implies $V(x, y, t) \leqslant \mathcal{E}_{\xi(\rho)}(g) \leqslant \delta$.

\subsection{Topological properties of the Skorokhod space used in Section 3}

Lemma 25. Given $t>0, D \subset \mathbb{R}^{d}$ a bounded domain, $\rho>0$ and the sets

$$
\begin{aligned}
& \mathcal{G}_{t}:=\left\{\Phi \in \mathbb{D}\left([0, t], \mathbb{R}^{d}\right) \mid \Phi(s) \in \overline{D \backslash B_{\rho}(0)} \text { for all } s \in[0, t]\right\}, \\
& \tilde{\mathcal{G}}_{t}:=\left\{\Phi \in \mathbb{D}\left([0, t], \mathbb{R}^{d}\right) \mid \Phi(s) \in \overline{D \backslash B_{\rho}(0)} \text { for all } s \in[0, t]\right. \\
&\text { except in a countable number of points }\},
\end{aligned}
$$

we have that $\tilde{\mathcal{G}}_{t}=\mathcal{G}_{t}$ and $\tilde{\mathcal{G}}_{t}$ is a closed set in $\mathbb{D}\left([0, t], \mathbb{R}^{d}\right)$ with respect to the Skorokhod topology.

Proof. 
Step 1: We prove that $\tilde{\mathcal{G}}_{t}$ is closed in $\mathbb{D}\left([0, t], \mathbb{R}^{d}\right)$ with respect to the Skorokhod topology. Let $\left(\Phi_{n}\right)_{n \in \mathbb{N}} \subset \tilde{\mathcal{G}}_{t}$ such that $d_{J_{1}}\left(\Phi_{n}, \Phi\right) \rightarrow 0$ as $n \rightarrow \infty$ for some $\Phi \in \mathbb{D}\left([0, t], \mathbb{R}^{d}\right)$. Let $\left(s_{k}\right)_{k \in \mathbb{N}}$ be the countable set of discontinuities of $\Phi$. For each $n \in \mathbb{N}$ we denote $\left(t_{k}^{n}\right)_{k \in \mathbb{N}}$ the countable set such that

$$
\Phi_{n}(s) \in \overline{D \backslash B_{\rho}(0)} \quad \text { for all } s \in[0, t] \backslash\left\{t_{k}^{n}\right\}_{k \in \mathbb{N}} .
$$

For all $s \in[0, t] \backslash\left(\bigcup_{n=1}^{\infty}\left\{t_{k}^{n}\right\}_{k \in \mathbb{N}} \cup\left\{s_{k}\right\}_{k \in \mathbb{N}}\right)$ it is a standard property of càdlàg functions (see [8], p.112) that

$$
\Phi_{n}(s) \rightarrow \Phi(s) \text { as } n \rightarrow \infty .
$$

Since $\overline{D \backslash B_{\rho}(0)}$ is a compact set of $\mathbb{R}^{d}, \Phi(s) \in \overline{D \backslash B_{\rho}(0)}$, which concludes the proof that $\tilde{\mathcal{G}}_{t}$ is closed in $\left(\mathbb{D}\left([0, t], \mathbb{R}^{d}\right), J_{1}\right)$.

Step 2: We prove that $\tilde{\mathcal{G}}_{t}=\mathcal{G}_{t}$. The inclusion $\tilde{\mathcal{G}}_{t} \supset \mathcal{G}_{t}$ is obvious. Let $\Phi \in \tilde{\mathcal{G}}_{t}$. If there exists $s \in[0, t]$ such that $\Phi(s) \notin \overline{D \backslash B_{\rho}(0)}$, by right-continuity of $\Phi$, there exists $\delta>0$ such that

$$
\Phi[s, s+\delta) \subset(\bar{D})^{c} \cup B_{\rho}(0)
$$

which violates $\Phi \in \tilde{\mathcal{G}}_{t}$.

Lemma 26. For any closed set $F \subset \mathcal{B}\left(\mathbb{R}^{d}\right)$ and $t>0$ we consider the following subset of $\mathbb{D}\left([0, t], \mathbb{R}^{d}\right)$

$$
\mathcal{A}:=\left\{\varphi \in \mathbb{D}\left([0, t], \mathbb{R}^{d}\right) \mid \varphi(s) \in F \quad \text { for some } s \in[0, t]\right\} .
$$

Then $\mathcal{A}$ is closed in $\mathbb{D}\left([0, t], \mathbb{R}^{d}\right)$ with respect to the Skorokhod topology.

Proof. Let $\left(\varphi_{n}\right)_{n \in \mathbb{N}}$ be a sequence of elements of $\mathcal{A}$ and $\varphi \in \mathbb{D}\left([0, t], \mathbb{R}^{d}\right)$ such that $d_{J_{1}}\left(\varphi_{n}, \varphi\right) \rightarrow 0$ as $n \rightarrow \infty$. For every $n \in \mathbb{N}$ let $s_{n} \in[0, t]$ such that $\varphi_{n}\left(s_{n}\right) \in F$. By right continuity of $\varphi_{n}$ there exists $\delta_{n}>0$ such that $\varphi_{n}\left(\left[s_{n}, s_{n}+\delta_{n}\right)\right) \subset F$. For every $n \in \mathbb{N}$ we denote $I_{n}:=\left[s_{n}, s_{n}+\delta_{n}\right)$. For every $n \in \mathbb{N}$ let $\left\{t_{n}^{k}\right\}_{k \in \mathbb{N}}$ be the set of discontinuities of $\varphi$ in $I_{n}$. Since for every $n \in \mathbb{N} \varphi_{n}$ and $\varphi$ are càdlàg functions we have

$$
\varphi_{n}(r) \rightarrow \varphi(r) \quad \text { for all } r \in \bigcup_{n \in \mathbb{N}}\left(I_{n} \backslash\left\{t_{n}^{k}\right\}_{k \in \mathbb{N}}\right) .
$$

Since $F$ is a closed subset of $\mathbb{R}^{d} \varphi(r) \in F$ for all $r \in \bigcup_{n \in \mathbb{N}}\left(I_{n} \backslash\left\{t_{n}^{k}\right\}_{k \in \mathbb{N}}\right)$. This proves that $\varphi \in \mathcal{A}$ and that $\mathcal{A}$ is closed in $\mathbb{D}\left([0, T], \mathbb{R}^{d}\right)$ with respect to the Skorokhod topology.

Acknowledgments The first author thanks Peter Imkeller (HU Berlin) and Sylvie Roelly (U. Potsdam) for the fruitful discussions on the subject. He also acknowledges the financial support from the project MASH(51099907) during his stay at U. Potsdam and from the FAPESP grant number 2018/06531-1 at UNICAMP-Campinas (SP). The second author would like to thank the School of Sciences at Universidad de los Andes for FAPA funding and MINCIENCIAS for travel funding in the framework of the Stic AMSUD 2019 Project "Stochastic analysis of non-Markovian phenomena". Both authors greatly acknowledge financial and infrastructure support by the DFG-funded International Research Training Group (IRTG) 1740 Berlin- São Paulo: Dynamical Phenomena in Complex Networks: Fundamentals and Applications hosted at Humboldt-Universität Berlin.

\section{References}

[1] A. de Acosta. Large deviations for vector-valued Lévy processes. Stoch. Proc. and Appl. vol. 51, 143-156 (1994)

[2] D. Applebaum. Lévy processes and stochastic calculus, volume 116 of Cambridge Studies in Advanced Mathematics. 
[3] Arrhenius, S. A. Über die Dissociationswärme und den Einfluß der Temperatur auf den Dissociationsgrad der Elektrolyte. Z. Phys. Chem. 4, 96-116 (1889) Doi: 10.1515/zpch-1889-0408. Cambridge University Press, second edition, (2009).

[4] N. Berglund. Kramers' law: Validity, derivations and generalisations, Markov Processes and Related Fields, 19 (3), 459-490 (2013)

[5] N. Berglund and B. Gentz. On the noise-induced passage through an unstable periodic orbit I: Two-level model, Journal of Statistical Physics, 114 (5-6) 1577-1618 (2004)

[6] N. Berglund and B. Gentz. The Eyring-Kramers law for potentials with nonquadratic saddles, Markov Processes and Related Fields, 3 (16) 549-598 (2010)

[7] C.-H. Rhee, J. Blanchet, B. Zwart. Sample path large deviations for Lévy processes and random walks with Regularly varying increments. eprint arXiv:1606.02795.

[8] P. Billinsgley. Convergence of Probability Measures. Wiley-Interscience 2nd edition (1999)

[9] F. Barret and A. Bovier and S. Méléard. Uniform estimates for metastable transitions in a coupled bistable system, Electronic Journal of Probability, 15, 323-345, 2010

[10] Bovier, A. and Eckhoff, M. and Gayrard, V. and Klein, M. Metastability and low lying spectra in reversible Markov chains, Communications in Mathematical Physics, 228, 219-255, 2002.

[11] A. Bovier, M. Eckhoff, V. Gayrard, M. Klein. Metastability in reversible diffusion processes I. Sharp asymptotics for capacities and exit times. J. Eur. Math. Soc., 6(4):399-424, (2004).

[12] A. A. Borovkov. Boundary-value problems for random walks and large deviations in function spaces. Theory Probab. Appl. vol. 12, 575-595 (1967) particles. Comm. in Math. Physics, vol. 56(2), 101113 (1977)

[13] A. Budhiraja, P. Dupuis, V. Maroulas. Variational representations for continuous time processes. Ann. de l'Inst. Henr. Poinc. (B) Probabilités et Statistiques. vol. 47(3), 725-747 (2011)

[14] A. Budhiraja, J. Chen, P. Dupuis. Large deviations for stochastic partial differential equations driven by a Poisson random measure. Stochastic Process. Appl, vol. 123(2), 523-560 (2013)

[15] A. Budhiraja, P. Nyquist. Large deviations for multidimensional state-dependent shot-noise processes. J. Appl. Probab. Vol. 52(4), pp. 1097-1114 (2015)

[16] A. Budhiraja. P. Dupuis. Analysis and Approximation of Rare Events. Representations and Weak Convergence Methods. Probability Theory and Stochastic Modelling Vol. 94. Springer (2019)

[17] Cerrai, S. and Roeckner, M., Large deviations for stochastic reaction-diffusion systems with multiplicative noise and non-Lipshitz reaction term Ann. Probab., 1B (32), 1100-1139, 2004.

[18] Cerrai, S., Debussche, A., Large deviations for the two-dimensional stochastic Navier-Stokes equation with vanishing noise correlation. Ann. Inst. Henri Poincaré Probab. Stat. 55 (2019), no. 1, 211-236.

[19] H. Cramér. Sur un nouveau théoreme-limite de la théorie des probabilités, Actualités scientiques et industrielles, Hermann et Cie, Paris. 736 (277) 2-23 (1938).

[20] A. Debussche, M. Högele, P. Imkeller. The dynamics of nonlinear reaction-diffusion equations with small Lévy noise. Springer Lect. Notes in Maths. vol. 2085 (2013)

[21] Deuschel, J.-D. and Stroock, D.,Large deviations, Pure and Applied Mathematics, Academic Press, 1989.

[22] A. Dembo, O. Zeitoni. Large Deviations Techniques and Applications. Applications of Mathematics vol. 38. Springer New York, 2nd ed. (1998)

[23] Donsker, M. D.; Varadhan, S. R. S. Large deviations for Markov processes and the asymptotic evaluation of certain Markov process expectations for large times. Probabilistic methods in differential equations (Proc. Conf., Univ. Victoria, Victoria, B. C., 1974), pp. 8288. Lecture Notes in Math., Vol. 451, Springer, Berlin, 1975. 
[24] P. Dupuis, R. S. Ellis. A Weak Convergence Approach to the Theory of Large Deviations. Wiley Series in Probability and Statistics. Wiley and Sons, New York (1997)

[25] K. Dzhaparidze, J.H. van Zantem. On Bernstein-type inequalities for martingales. Stoch. Proc. Appl. 93, pp 109-117 (2001)

[26] Eyring, H., The activated complex in chemical reactions, "The Journal of Chemical Physics, 3, 107-115 (1935)

[27] D. Florens, H. Pham. Large deviations probabilities in estimation of Poisson random measures. Stoch. Proc. and Appl, vol. 76, 117-139 (1998)

[28] Ventsel', A. D. and Freidlin, M. I., On small random perturbations of dynamical systems, Russian Mathematical Surveys, 25 (1) 1-55, 1970.

[29] M. I. Freidlin, A. D. Wentzell. Random Perturbations of Dynamical Systems. Grundlehren der Mathematischen Wissenschaften 260. Springer New York, 2nd ed. (1998)

[30] M. I. Freidlin. Quasideterministic approximation, metastability and stochastic ressonance. Physics D 137, pp. 333-352 (2000)

[31] Gamboa, F. and Nagel, J. and Rouault, A., Sum rules and large deviations for spectral matrix measures. Bernoulli 25 (2019), no. 1, 712-741.

[32] Godovanchuk, V. V., Asymptotic probabilities of large deviations due to large jumps of a Markov process, Theory of Probability and its Applications, 26, 314-327 (1982)

[33] Godovanchuk, V. V., Probabilities of large deviations for sums of independent random variables attracted to a stable law, Theory of Probability and its Applications, 23, 603-608 (1979)

[34] M. Högele and I. Pavlyukevich. The exit problem from a neighborhood of the global attractor for dynamical systems perturbed by heavy-tailed Lévy processes Journal of Stochastic Analysis and Applications, 32(1), 163-190 (2013)

[35] N. Ikeda and S. Watanabe. Stochastic Differential Equations and Diffusion Processes. North-Holland Publishing Co., Amsterdam, (1981)

[36] P. Imkeller, I. Pavlyukevich. First exit times of SDEs driven by stable Lévy processes. Stoch. Proc. Appl. vol. 116(4), 611-642 (2006)

[37] P. Imkeller, I. Pavlyukevich. Meta-stable behavior of small noise Lévy-driven diffusions. ESAIM Probab. Stat. 12, 412-437 (2008)

[38] P. Imkeller, I. Pavlyukevich, T. Wetzel. First exit times for Lévy-driven diffusions with exponentially light jumps. Ann. Probab. 37, no. 2, 530-564 (2009)

[39] Imkeller, P. and Pavlyukevich, I. and Wetzel, T., The hierarchy of exit times of Lévy-driven Langevin equations, The European Physical Journal Special Topics, 191, 211-222 (2010)

[40] J. Jacod, A.N. Shiryaev. Limit Theorems for Stochastic Processes. Springer-Verlag (1987)

[41] G. Kallianpur, J. Xiong. Stochastic Differential Equations in Infnite Dimensional Spaces. Inst. Math. Stats. Lecture Notes - Monograph Series, Vol. 26 (1995)

[42] Kramers, H. A., Brownian motion in a field of force and the diffusion model of chemical reactions, Physica, 7, 284-304 (1940)

[43] H. Kunita. Stochastic diferential equations based on Lévy processes and stochastic fows of difeomorphisms. Real and Stoch. Anal. ed. by M. M. Rao, 305-373, Birkhauser (2004)

[44] C. Leonard. Large deviations for Poisson random measures. Stoch. Proc. and Appl. vol. 85, 93-121 (2000)

[45] Logachov, A., Logachova, O., Yambartsev, A., Large deviations in a population dynamics with catastrophes. Statist. Probab. Lett. 149 (2019), 29-37 
[46] Löcherbach, E. Large deviations for cascades of diffusions arising in oscillating systems of interacting Hawkes processes. J. Theoret. Probab. 32 (2019), no. 1, 131162

[47] J. Lynch, J. Sethuraman. Large deviations for processes with independent increments. Ann. Probab. 15, no.2, 610-627 (1987)

[48] V. Maroulas. Large Deviations for Infinite Dimensional System with Jumps. Mathematika 57, pp. 175192 (2011)

[49] I. Pavlyukevich. First exit times of solutions of stochastic diferential equations driven by multiplicative Lévy noise with heavy tails. Stoch. and Dyn. vol.11(2-3), 495-519 (2011)

[50] A. Puhalskii. Large deviations of semimartingales via convergence of the predictable characteristics. Stochastics and Stochastic Reports, 49(1-2), pp. 27-85 (1994)

[51] C. Robinson. Dynamical Systems. Stability, Symbolic Dynamics, and Chaos. Second Edition. CRC Press.

[52] J. Rosiński. Tempering stable processes. Stoch. Proc. and Appl. vol. 177(6), pp. 677-707 (2007)

[53] K. Sato. Lévy Processes and Infnitely Divisible Distributions. Cambridge Studies in Advanced Mathematics 2nd ed. (2013)

[54] M. Salins, K. Spiliopoulos. Metastability and exit problems for systems of stochastic reaction-diffusion equations. (2019) https://arxiv.org/pdf/1903.06038.pdf.

[55] W. Siegert. Local Lyapunov Exponents: Sublimiting Growth Rates of Linear Random Differential Equations, Lecture notes in Mathematics, vol. 1961, Springer Verlag (2009)

[56] S.R.S. Varadhan. Large Deviations and Applications. CBMS-NSF Regional Conference Series in Applied Mathematics vol.46, Soc. for Ind. and Appl. Math. (1984)

[57] Shi, W. A note on large deviation probabilities for empirical distribution of branching random walks. Statist. Probab. Lett. 147 (2019), 18-28.

[58] Vent-tsel', A. D., On the asymptotic behavior of the first eigenvalue of a second-order differential operator with small parameter in higher derivatives, Theory of Probability \&amp; Its Applications, 20 (3), 599602, http://dx.doi.org/10.1137/1120064, DOI: 10.1137/1120064 (1976).

[59] Wentzell, A. D. Limit theorems on large deviations for Markov stochastic processes. Translated from the Russian. Mathematics and its Applications (Soviet Series), 38. Kluwer Academic Publishers Group, Dordrecht, 1990. xvi+176 pp. ISBN: 0-7923-0143-9

[60] X. Yang, J. Zhai, T. Zhang. Large deviations for SPDEs of jump type. Stochastics and Dynamics Vol. 15, No. 04, 1550026 (2015). https://doi.org/10.1142/S0219493715500264

[61] J. Zhai, T. Zhang. Large deviations for 2-D stochastic NavierStokes equations driven by multiplicative Lévy noises. Bernoulli Volume 21, Number 4 (2015), 2351-2392. 